Review

\title{
Evolution of Macromolecular Docking Techniques: The Case Study of Nickel and Iron Metabolism in Pathogenic Bacteria
}

\author{
Francesco Musiani * and Stefano Ciurli * \\ Laboratory of Bioinorganic Chemistry, Department of Pharmacy and Biotechnology, \\ University of Bologna, Viale G. Fanin 40, Bologna I-40127, Italy \\ * Authors to whom correspondence should be addressed; \\ E-Mails: francesco.musiani@unibo.it (F.M.); stefano.ciurli@unibo.it (S.C.); \\ Tel.: +39-051-2096236 (F.M.); +39-051-2096204 (S.C.); Fax: +39-051-2096203 (F.M. \& S.C.).
}

Academic Editor: Rino Ragno

Received: 28 April 2015 / Accepted: 28 July 2015 / Published: 5 August 2015

\begin{abstract}
The interaction between macromolecules is a fundamental aspect of most biological processes. The computational techniques used to study protein-protein and protein-nucleic acid interactions have evolved in the last few years because of the development of new algorithms that allow the a priori incorporation, in the docking process, of experimentally derived information, together with the possibility of accounting for the flexibility of the interacting molecules. Here we review the results and the evolution of the techniques used to study the interaction between metallo-proteins and DNA operators, all involved in the nickel and iron metabolism of pathogenic bacteria, focusing in particular on Helicobacter pylori $(\mathrm{Hp})$. In the first part of the article we discuss the methods used to calculate the structure of complexes of proteins involved in the activation of the nickel-dependent enzyme urease. In the second part of the article, we concentrate on two applications of protein-DNA docking conducted on the transcription factors $H p F u r$ (ferric uptake regulator) and $H p N i k R$ (nickel regulator). In both cases we discuss the technical expedients used to take into account the conformational variability of the multi-domain proteins involved in the calculations.
\end{abstract}

Keywords: macromolecular docking; urease; urease metallocenter biosynthesis; NikR; Fur; Helicobacter pylori 


\section{Introduction}

Living organisms rely on protein-protein and on protein-nucleic acid interactions to perform their functions [1-4]. Considering only protein-protein binary interactions, this number can go from $\mathrm{ca}$. 10,000 in Escherichia coli [5], to ca. 18,000 in higher plants of the Arabidopsis genus [6], to 150,000-500,000 in human cells [7,8]. Despite their evident importance, the interactions between macromolecules are not fully understood at the structural level. Indeed, only a fraction of the putatively analyzable unique protein-protein interfaces are so far available from high-resolution X-ray crystallography and nuclear magnetic resonance (NMR) spectroscopy [9]. Structural information can also be obtained from low-resolution experimental techniques, such as cryo-electron microscopy or small-angle X-ray scattering which, however, do not provide enough molecular details of the interacting surfaces [10]. Experimental techniques can thus be complemented by computational docking methods aimed to model the quaternary structure of complexes formed by two or more interacting macromolecules. Protein-protein complexes are the most commonly-attempted targets of such modelling [10-15], followed by protein-nucleic acid complexes [16-18]. In the classical (or ab initio) docking methods, the calculation itself only produces plausible candidate structures. These candidates must then be ranked a posteriori using scoring functions or validation/filtering procedures that use experimental data to identify structures that are most likely to occur in nature. In recent years, integrative and information-driven algorithms changed the classical ab initio docking procedure by encoding a priori information derived from experimentally-identified or predicted protein interfaces to drive the docking process [10].

Here we review and discuss the evolution of the techniques used by us and by other groups to study the interactions between macromolecules in two macro-test cases: (i) the prediction of the complexes formed by the accessory proteins involved in the activation of the nickel-dependent enzyme urease; and (ii) the prediction of protein-DNA complexes involving two bacterial transcriptional factor, namely the ferric uptake regulator (Fur) and the nickel regulator NikR, involved in cellular iron and nickel metabolism.

\section{Macromolecular Docking Overview}

Macromolecular docking is usually defined as the structural prediction of a molecular complex starting from the separated structures of its members. A typical docking algorithm involves three steps (see Scheme 1). The first step consists of the exploration of the large number of possible orientations and conformations that the members of a complex can assume in tridimensional space. This is usually done through a "search algorithm". This exploration step is then followed by a scoring step of the resulting model complexes using an appropriate criterion. Finally, the procedure is often concluded by the refinement of a restricted number of the obtained structures of the complex.

\subsection{Search Algorithms}

In typical docking algorithms, one molecule is fixed (the so-called "static molecule") and the other molecule is translated and rotated around (the so-called "moving molecule"). Depending on the search strategies, current ab initio docking programs can be divided into three general categories: (i) exhaustive global search; (ii) local shape feature matching; and (iii) randomized search algorithms [14]. 


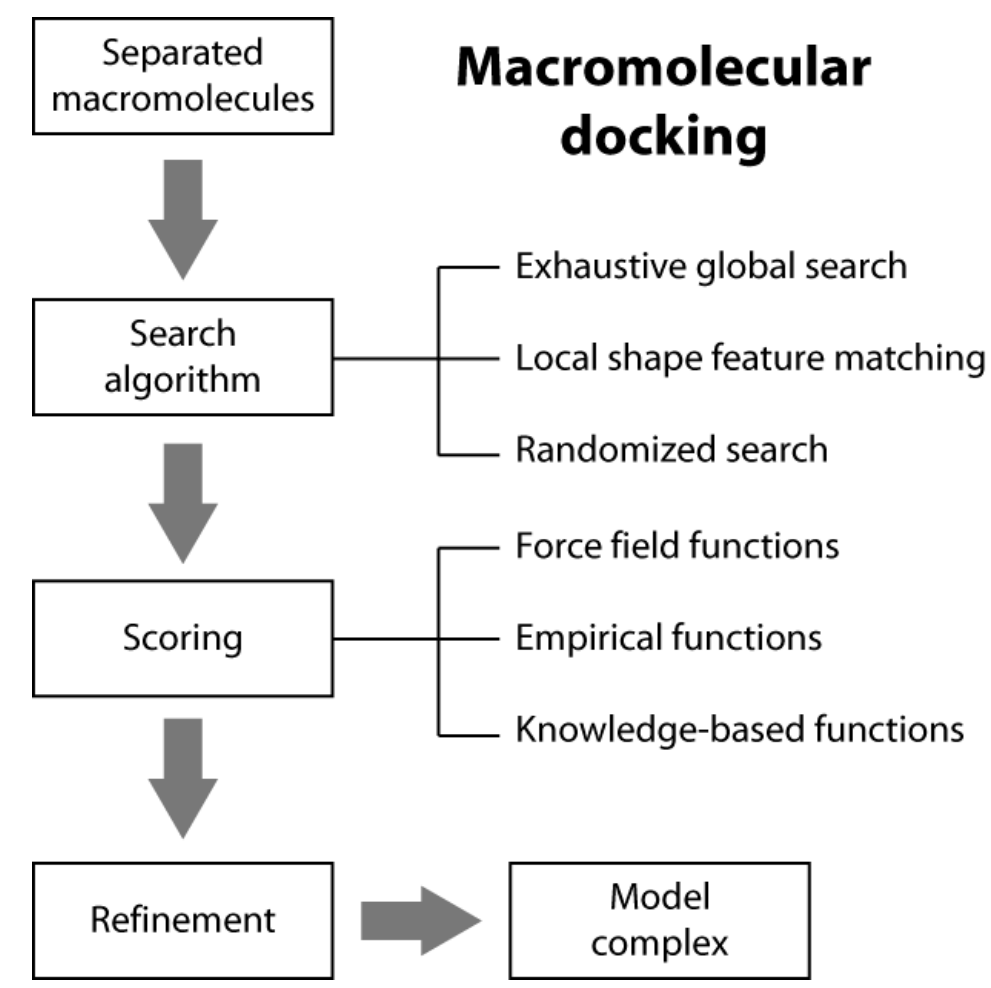

Scheme 1. Macromolecular docking flow-chart.

The global search algorithms perform a scan over the three rotational and the three translational degrees of freedom. Given the huge number of possible orientations to be sampled, it is computationally prohibitive to evaluate all the binding complexes using a standard search method. To reduce the computational cost, two types of approaches have been developed for this type of exhaustive global search: fast Fourier transform (FFT) correlation and direct search algorithms [14]. The FFT-based algorithm accelerates the search process in the translational space by moving to the imaginary space through several FFT calculations. In the direct search algorithm approach, the molecular shape of two interacting molecules is first mapped onto a tridimensional grid and the shape matching is then directly performed in the Cartesian grid space to find the geometric fit between the two molecules.

In local shape feature-matching algorithms, the interacting molecules are represented by their shapes, such as the molecular surface. The search algorithms find those matches that give a good local shape complementarity between the two molecules. Local shape feature-matching algorithms also tend to generate more binding orientations towards those sites with good shape complementarity. Therefore, a clustering step is often necessary after the docking calculation to remove the redundancy in the final solutions [14].

In randomized search algorithms, the moving molecule is randomly placed around the binding site for a local search or around the whole static molecule for a global search based on a certain number of rules. The placement procedure can be improved by using additional information, such as the molecular surfaces, to generate more realistic initial binding orientations. Then, from their starting positions, each of the initially generated orientations is optimized and/or refined through a multistage sampling and/or multiscale modeling approach that use stochastic algorithms, such as genetic algorithms and/or Monte Carlo methods [14]. 
Docking search algorithms can use information extracted from experimental or bioinformatics sources to guide the formation of the complex. In this way, the sampling can be limited to the regions defined by the data and the number of unrealistic solutions can be dramatically reduced. On the other hand, the inclusion of experimental/prediction data does not guarantee that all complexes are correct [10].

\subsection{Scoring Functions}

There are three general classes of scoring functions: (i) force field; (ii) empirical; and (iii) knowledge-based, even if some algorithms use a combination of two or all of them [19].

In the case of force field scoring functions, the interaction affinities are estimated by considering physical atomic interactions, such as van der Waals and electrostatic interactions and bond stretching/ bending/torsional forces [19]. Force field functions and parameters are usually derived from both experimental data and quantum mechanical calculations. Because the binding of the molecules normally takes place in the presence of water, the desolvation energies of the interacting molecules are sometimes taken into account using implicit solvation methods [19].

Empirical scoring functions estimate the binding affinity of a complex on the basis of a set of weighted energy terms [19]. The energy coefficients are determined by fitting the binding affinity data of a training set of protein-ligand complexes with known three-dimensional structures. Compared to the force field scoring functions, the empirical scoring functions are much faster in binding score calculations due to their simple energy terms.

The knowledge-based (also known as statistical potentials) scoring functions are based on statistical observations of intermolecular close contacts in structural databases that are used to derive "potentials of mean force". This method is based on the assumption that close intermolecular interactions between certain types of atoms or functional groups that occur more frequently than one would expect by a random distribution are likely to be energetically favorable and therefore contribute favorably to binding affinity [20].

\subsection{A Peculiar Case: Protein-DNA Docking}

DNA is considered a difficult target for macromolecular docking because of its inherent flexibility summed with the scarcity of the information to define the DNA-binding interfaces. DNA can exhibit large conformational changes upon binding to a protein, which can greatly modify the shape of the interaction surface. As a direct consequence, the total conformational space that needs to be searched in order to find favorable conformations becomes too computationally expensive [21]. In the present review we discuss protein-DNA calculations performed using the program Haddock [22,23] and a two-stage docking approach specifically developed for the calculation of protein-DNA complexes [21]. These calculations yielded good performances [24] and are described in the examples discussed here. Haddock (High Ambiguity Driven biomolecular DOCKing) implements an approach that uses biochemical and/or biophysical interaction data to drive the docking process. The calculation is guided by defining selected residues as "active" in the protein-protein or protein-DNA interaction. The docking algorithm rewards the complexes that have these active residues on the interaction interface. Haddock simulations are composed of three rounds: (i) a rigid body energy minimization that produces a user-defined number of putative docking complexes (usually 1000); (ii) a semi-flexible simulated annealing carried out on the 
best solutions calculated in the first round and found on the basis of the intermolecular energy (habitually 200); and (iii) an explicit water refinement carried out on the same structures of the previous step. Haddock has been implemented by using the program CNS [25] for structure calculations and python scripts derived from ARIA [26] for automation. The solutions are then clustered on the basis of the pairwise backbone RMSD and further analyzed for structural and functional congruence. The cut-off for the clustering of protein-protein docking solution is typically set to $7.5 \AA$. The protein-DNA docking protocol developed for Haddock starts with the generation of a model for the unbound DNA operator using the DNA analysis and rebuilding software 3DNA implemented in the 3D-DART server [27]. In the first docking round, additional restraints are introduced for the DNA fragment to maintain base planarity and Watson-Crick bonds. Subsequently, the DNA conformation in the docked resulting structures are analyzed using the program DART [27] in order to determine trends in DNA bending and twisting, a type of information that is used to generate an ensemble of custom DNA models representing the accessible conformations. A second, and final, docking round is then carried out following the same approach described for the first round, but this time including the ensemble of pre-bent DNA models generated above and representing various degrees of conformational flexibility of the nucleic acid. In this round, the conformational freedom of the DNA molecule is restricted at the semi-flexible refinement stage (see below) in order to prevent helical deformation.

Here below we describe and discuss specific examples of macromolecular docking applied to proteins involved in the cellular metabolism of nickel and iron.

\section{Urease Activation}

Urease is a nickel dependent enzyme that catalyzes urea hydrolysis in the last step of organic nitrogen mineralization to give ammonia and carbamate, which spontaneously decomposes to give a second molecule of ammonia and bicarbonate. The reaction products cause an overall $\mathrm{pH}$ increase that has negative effects both on human and animal health, as well as on the ecosphere [28,29]. The structures of urease from several bacteria and from higher plants have revealed the molecular architecture of the enzyme (Figure 1A) [28,29]. In the fully-conserved urease active site, two Ni(II) ions are bridged by the oxygen atoms of a carbamylated lysine residue and bound to two histidines. One $\mathrm{Ni}$ (II) ion is additionally bound to an aspartate carboxylate oxygen. The coordination geometry of the $\mathrm{Ni}$ (II) ions is completed by a water molecule bound to each metal ion and by a nickel-bridging hydroxide ion (Figure 1A) [28,29]. In vivo, holo-urease is post-translationally synthesized starting from apo-urease and following an activation process that involves $\mathrm{CO}_{2}$ uptake for lysine carbamylation, hydrolysis of GTP, and $\mathrm{Ni}$ (II) delivery into its active site. These events are typically carried out by four specific accessory proteins named UreD, UreF, UreG, and UreE [28]. The "classical" hypothesis for this process [28,30] involves the sequential binding of UreD, UreF, and UreG [or of a preformed aggregate of UreD, UreF, and UreG (UreDFG)] to obtain a pre-activation complex that carbamylates the active site lysine side chain and further binds $\mathrm{Ni}(\mathrm{II})$ ions delivered by UreE $[28,30]$ through a route driven by GTP hydrolysis (Figure 1B, blue arrows path). Little is known on the functional properties of UreD [31], apparently the first protein that binds apo-urease. UreF is proposed to bind the urease-UreD complex through a direct interaction with UreD [32] and favors the formation of the urease-UreDFG complex [33]. UreG is responsible for coupling GTP hydrolysis to the process of urease activation and it is proposed to catalyze, in the presence 
of $\mathrm{CO}_{2}$, the formation of carboxyphosphate, an excellent carbamylation agent for the metal-binding lysine in the urease active site [33]. UreG is the first case of an intrinsically disordered enzyme [34], which can retain enzymatic activity owing to the rigidity of the active-site environment [35]. UreF has been also proposed to regulate the function and the folding of UreG, acting as a GTPase-activating protein (GAP) [36].
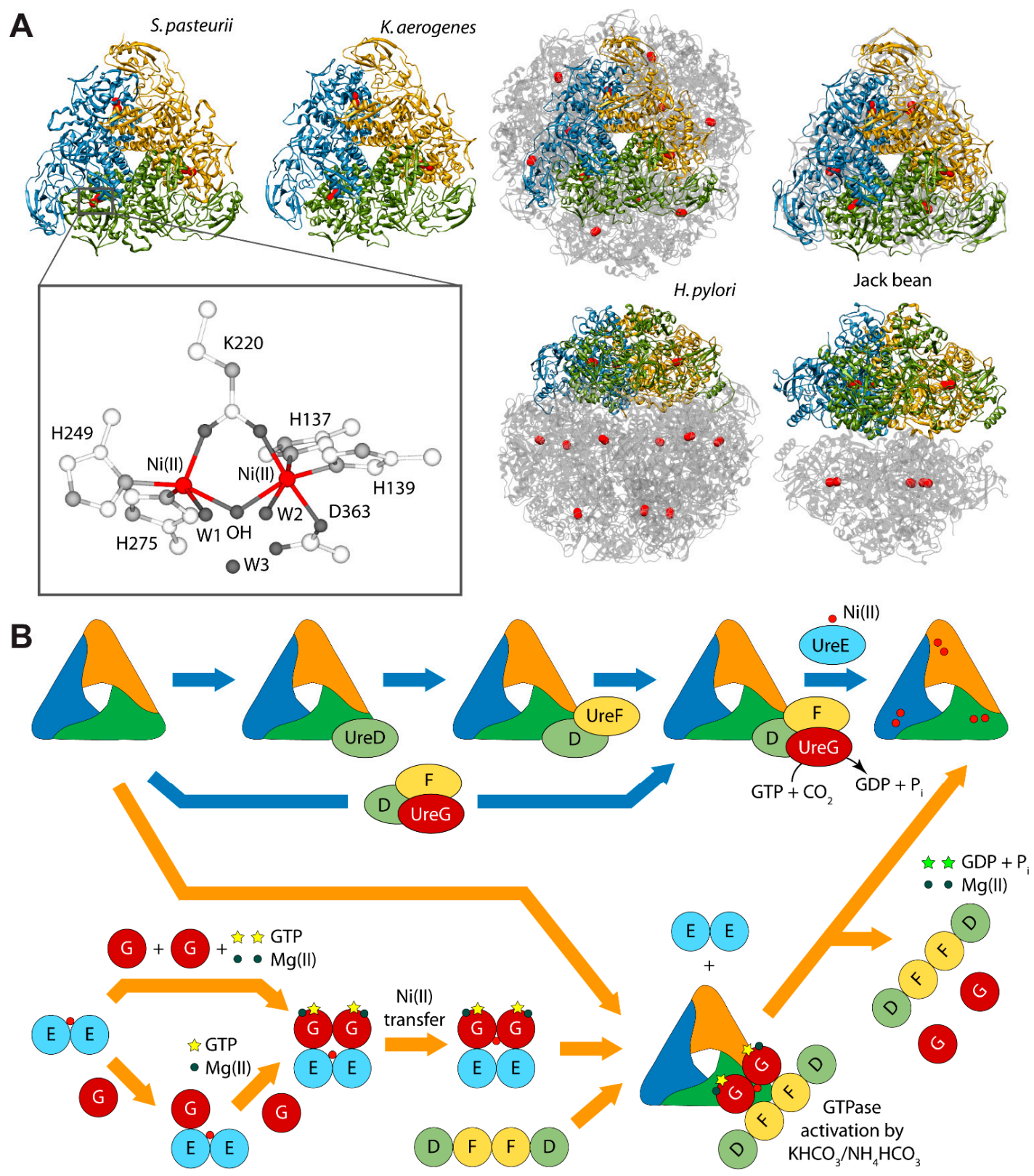

Figure 1. (A) Ribbon diagram of urease from S. pasteurii, K. aerogenes, H. pylori, and jack bean. Ribbons are colored to evidence the chains composing the protomers. Ni(II) ions are reported as red spheres. The proteins are seen through the ternary axis (top panels). The bottom panels are rotated by $90^{\circ}$ around the horizontal axis $v s$. the top panels. The inset reports the details of $\mathrm{Ni}$ (II) coordination geometry of in native urease active site; (B) Schematic representation of the proposed mechanisms for urease activation. 
Finally, UreE is proposed to be the metallo-chaperone in charge of delivering Ni(II) ions [37], and actively transferring $\mathrm{Ni}$ (II) to urease apo-protein within the apourease-UreDFG complex in a GTP-dependent activation process [38]. Indeed, in vivo studies using yeast two-hybrid analysis [39,40] and coimmunoprecipitation assays [40], as well as calorimetry and NMR spectroscopy [41] indicated a direct interaction between UreE and UreG from Helicobacter pylori $(\mathrm{Hp})$.

Even though the structure of urease bound to any of the accessory proteins is not yet available, the crystal structure of the (UreF) $)_{2}$ homodimer [42] and the structure of the (UreDF) 2 [43] and (UreDFG) $)_{2}$ [44] complexes from $H$. pylori have been recently reported (Figure $2 \mathrm{~A}$ ). The crystal structure of the $H p(\mathrm{UreDFG})_{2}$ complex contains two copies of each of UreF, UreD, and UreG, related by two-fold symmetry, forming a dimer of heterotrimers (Figure 2A). HpUreF adopts an all-helical fold that consists of a bundle of nine $\alpha$-helices arranged in an antiparallel fashion. The UreF dimerization occurs through $\alpha$-helices 2, 3, 8 and 9 [42]. $H p$ UreD fold consists of $17 \beta$-strands and two $\alpha$-helices located near the $C$-terminus. The structure is dominated by two mixed strand $\beta$-sheets [43]. The topology of $H p U r e G$ is characteristic of the SIMIBI class GTPases [45] and the protein was co-crystalized with one GDP molecule per monomer. The sequences of UreG feature a Cys-Pro-His $(\mathrm{CPH})$ conserved motif that is fundamental for the metal binding properties of the protein $[41,46]$. In the $H p(\mathrm{UreDFG})_{2}$ structure (as well in previously calculated model structures [47,48]) the CPH motif is found in a cleft at the UreG dimerization interface (Figure 2A) [44]. Interestingly, despite the fact that $S p \mathrm{UreG}$ and $H p \mathrm{UreG}$ model structures previously published by our group were based on distant homologues [34,47-49], the experimental structure of $H p(\mathrm{UreG})_{2}$ found in the $H p(\mathrm{UreDFG})_{2}$ complex and the model structure of dimeric $H p \mathrm{UreG}$ are very similar (Figure 2B), with a root mean square deviation (RMSD) of only $1.8 \AA$ for the $\mathrm{C} \alpha$ atoms. Structural information on UreE proteins from various bacteria has been derived from numerous crystallographic studies: UreE from Sporosarcina pasteurii (formerly known as Bacillus pasteurii, SpUreE) [50,51], Klebsiella aerogenes (KaUreE) [52], and H. pylori (HpUreE) [53,54] display a similar fold made by a symmetric homo-dimer, with each monomer composed of two domains connected by flexible linkers (Figure 2C). The fully conserved metal binding site is located on the surface of the $C$-terminal domain, at the dimerization interface. The $N$-terminal domain has been found in slightly different orientations with respect to the $C$-terminal domain, suggesting that some inter-domain conformational freedom is available for this protein, a feature possibly related to induced-fit processes that occur during the formation of protein-protein complexes involving the other urease chaperones [55]. On the other hand, this means that the UreE $\mathrm{N}$-terminal domain conformations observed in the solid state could not be the same needed for the correct formation of the complex with UreG [41]. The entirety of the structural information from crystallography, together with UV-VIS spectroscopy, light scattering experiments, and GTPase activity assays performed on $\mathrm{HpUreG}$, recently suggested a new mechanism for the biosynthesis of the urease active site (Figure 1B, orange arrows path) [56]. In the new pathway, the Ni(II)-bound UreE dimer binds two apo-UreG monomers, facilitating GTP uptake by UreG in the presence of $\mathrm{Mg}(\mathrm{II})$ ions. The UreG binding to UreE can, in principle, occur either in a single or in a multistep process. In the (UreEG)2 complex, the Ni(II) ion is then translocated from UreE to UreG. Subsequently, the pre-formed (UreDF) 2 complex competes with UreE for the $\mathrm{Ni}(\mathrm{II})-(\mathrm{UreG})_{2}$ dimer to form the supercomplex apo-urease/Ni(II)-(UreDFG)2. Finally, the GTP hydrolysis performed by UreG is catalyzed by $\mathrm{KHCO}_{3} / \mathrm{NH}_{4} \mathrm{HCO}_{3}$ to complete the nickel insertion into the apo-urease. 


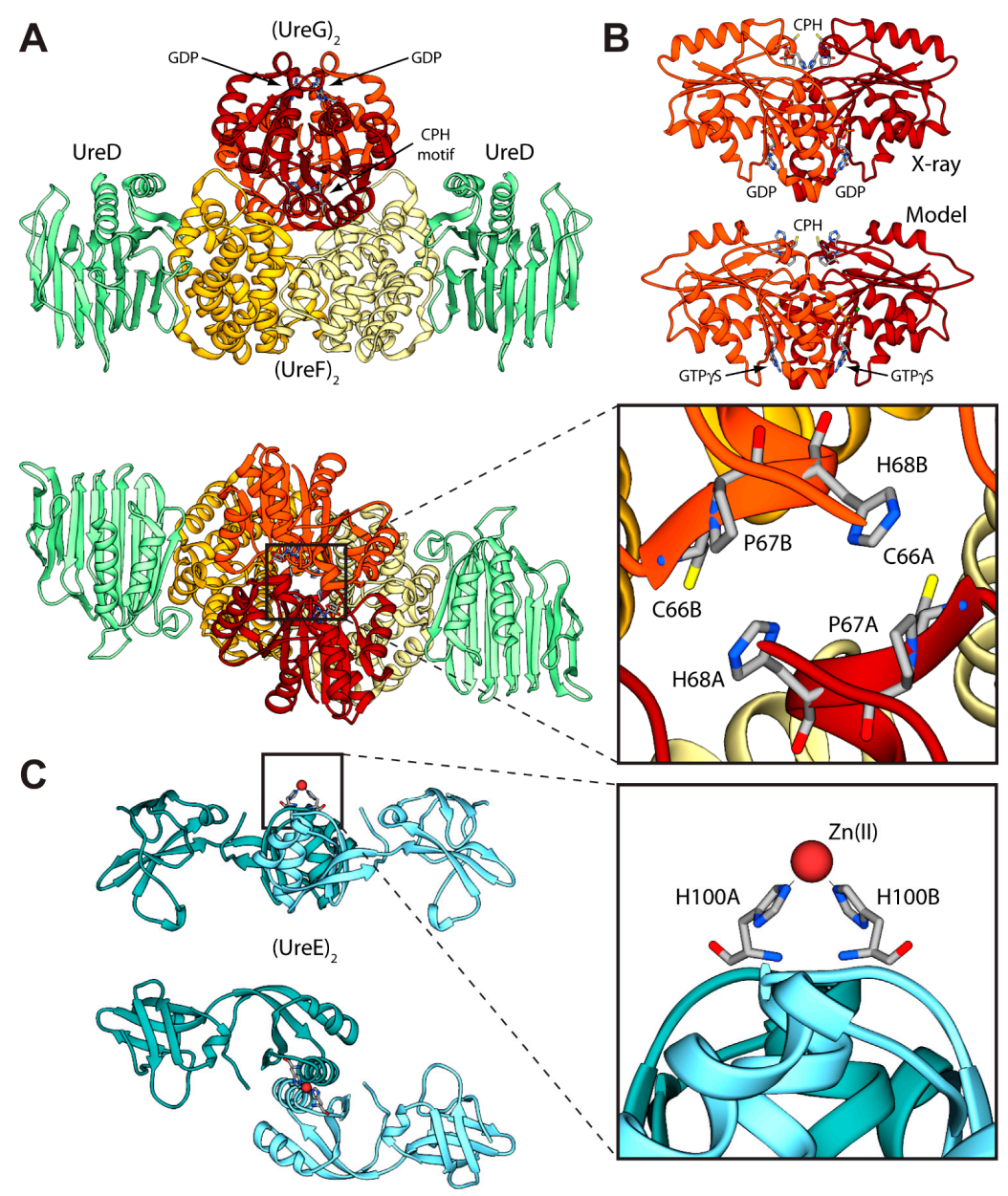

Figure 2. (A) Ribbon diagram of the (UreDFG) 2 crystal structure from H. pylori (PDB id 4HI0) [44]. $H p \mathrm{UreD}, H p \mathrm{UreF}$, and $H p \mathrm{UreG}$ chains are colored in light green, yellow and dark red, respectively, with the darker and lighter portions highlighting the different monomers. The views in the top and bottom panel are rotated by $90^{\circ}$ along the horizontal axis. The inset reports the position of the residues forming the HpUreG Cys-Pro-His conserved motif; (B) Comparison between the $H p U r e G$ dimeric crystal structure, as found in the $H p(\mathrm{UreDFG})_{2}$ complex, and the homology model structure calculated in ref. [47]; (C) Ribbon scheme of the structure of $S p U$ reE homodimer (PDB id: 1EAR); the two monomers are colored in light blue, with the darker and lighter portions representing the two monomers. The views in the top and bottom panel are rotated by $90^{\circ}$ along the horizontal axis. In the inset is reported the detail of the $S p U$ reE metal binding site.

\subsection{Calculation of the Structure of the UreEG Complex}

The first attempt to model the UreE-UreG complex from H. pylori (Hp(UreEG)) was published in 2009 [41]. The calculation was prompted by a number of experimental observations: (i) $S p U r e G$ and Mycobacterium tuberculosis $\mathrm{UreG}$ (Mt UreG) are dimers in native conditions, while $\mathrm{KaUreG}$ and $H p \mathrm{UreG}$ are monomeric proteins [47]; (ii) $\mathrm{Zn}$ (II)-driven protein dimerization occurs in vitro for $H p \mathrm{UreG}$, with one $\mathrm{Zn}$ (II) ion binding at the protein dimerization interface using the conserved cysteine and histidine residues from the $\mathrm{CPH}$ motif from each monomer [47]; (iii) $\mathrm{Zn}$ (II) and $\mathrm{Ni}$ (II) share the same binding site at the interface of the protein dimer in the $S p$ UreE crystal structure [50], with $\mathrm{Zn}$ (II) 
affinity ten times lower than $\mathrm{Ni}$ (II)-affinity for $\mathrm{KaUreE}$ [57], while the thermodynamics of Zn(II) binding to $H p$ UreE is very similar to that of $\mathrm{Ni}(\mathrm{II})$ [41]; (iv) the $H p$ UreEG protein complex contains two monomers of $H p \mathrm{UreG}$ dimerized onto one $H p \mathrm{UreE}$ dimer [41], with an interaction stabilized by the presence of $\mathrm{Zn}(\mathrm{II})$ and not by $\mathrm{Ni}$ (II) [41], suggesting a role for $\mathrm{Zn}$ (II) in promoting the UreE interaction network. Thus, the hypothesis at the basis of the docking calculation was that the two conserved metal binding sites found on dimeric UreE and UreG are able to come in close contact during the formation of the $H p(\text { UreEG })_{2}$ complex. The only UreE-available structures at that time were those from S. pasteurii and $K$. aerogenes, while there was no structure for UreG from any organism. Thus, the structures of $H p U r e E$ and $H p U r e G$ were modelled starting from homologue experimental structure [41]. In the case of $H p U r e E$ the templates were the crystal structures of $S p \mathrm{UreE}$ and $\mathrm{KaUreE}$ (PDB id: 1EAR [50] and 1GMW [52], respectively) following a previously established procedure [55], while for $H p$ UreG the template structure was HypB from Methanocaldococcus jannaschii (MjHypB, PDB id: 2HF8 [58]), an homologue GTPase involved in the biosynthesis of [Ni,Fe]-hydrogenase [47]. The RosettaDock software [59] was used to calculate an initial complex between the model structure of dimeric $H p \mathrm{UreG}$, and the central $C$-terminal domains of dimeric $H p U r e E$. This step allowed the calculations of a complex not biased by the conformation of the $\mathrm{HpUreE} \mathrm{N}$-terminal domains. The protocol used by RosettaDock included (i) prepacking of the partners to remove clashes in the free monomers; (ii) global search of rigid-body orientations; and (iii) clustering of the low-energy models and selection of the largest cluster as prediction. The global search step starts from a large number of random initial orientations and brings the partners into glancing contact and removes clashes. It then proceeds by optimizing rigid-body orientation at a low-resolution level (where each amino acid side-chain is represented by a centroid pseudo-atom that is positioned according to an average position determined from a set of known structures from the PDB), and subsequently builds initial side-chain conformations using a Monte Carlo search through a backbone-dependent rotamer library. Finally, the protocol uses $c a$. 50 cycles of Monte Carlo minimization to optimize the side-chain and rigid-body degrees of freedom using a free energy function dominated by short-range Lennard-Jones and hydrogen-bonding interactions, and an implicit solvation model [59]. A search of 1000 complexes was carried out by randomly translating and rotating the initial positions of the interacting proteins. The complex with the best RosettaDock score was selected among all generated models for the subsequent refining cycle, carried out by applying 1000 times a perturbation to the starting structure. The $\mathrm{C} \alpha$ trace of this complex and the crystal structures of $M j \mathrm{HypB}, S p \mathrm{UreE}$, and $\mathrm{KaUreE}$ were used as templates to build 200 structural models of the $H p(\mathrm{UreEG})_{2}$ complex using the Modeller software [60]. The best model was selected on the basis of the lowest value of the Modeller objective function. This modelling step allowed the addition of the $H p \mathrm{UreE} N$-terminal domains to the structure of the calculated model complex between the $C$-terminal domain of $H p \mathrm{UreE}$ and $H p \mathrm{UreG}$. The final $H p(\mathrm{UreEG})_{2}$ model (Figure $3 \mathrm{~A}$ ) showed the two proteins facing each other along their extended axes, and only limited modifications of the proteins backbone, restricted both in extent and in topology distribution, were necessary in order to optimize the docking procedure. The central pocket formed on the $H p U r e G$ surface around the conserved $\mathrm{CPH}$ motif matches the shape and volume of the protruding metal binding region on the surface of $H p U$ reE. The shallow crevice formed between the central $C$-terminal domain and the peripheral $N$-terminal domain of $H p \mathrm{UreE}$ is filled with the bulge found on the surface of $H p \mathrm{UreG}$ around the rim of the protein dimerization interface, and an overall continuous contact surface, with optimal electrostatic complementarity, was obtained (Figure 3B) [41]. 
A

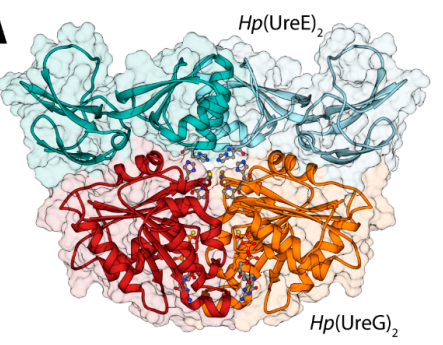

B
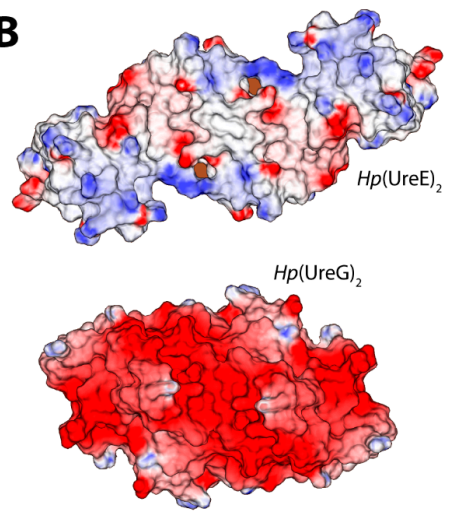

E

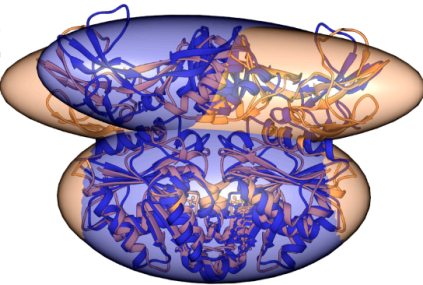

$\mathbf{F}$

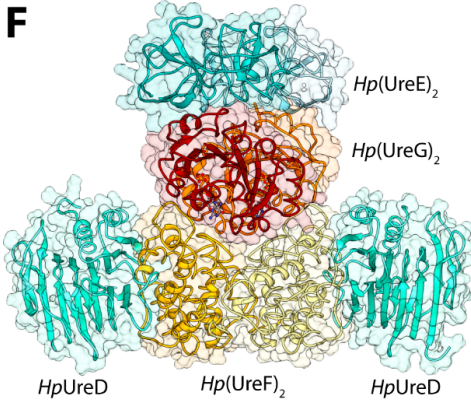

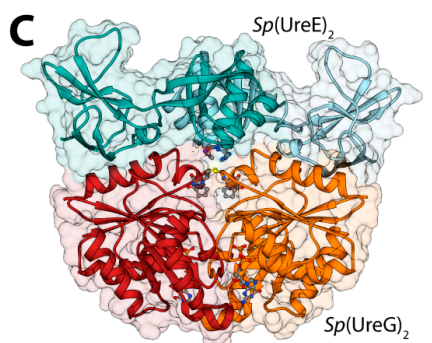

D
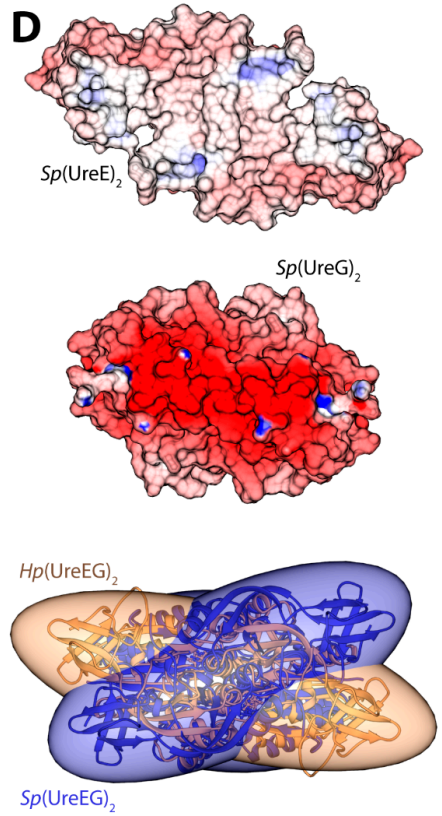

$S p\left(\right.$ UreEG) ${ }_{2}$

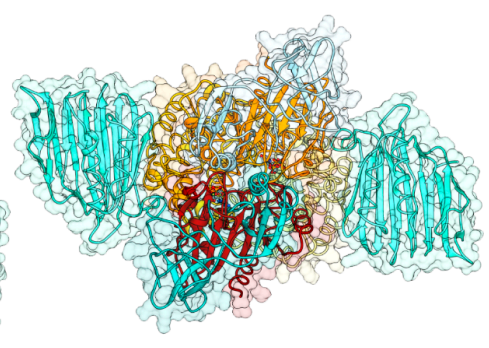

Figure 3. (A,C) Ribbon schemes and solvent-excluded surfaces of the model complexes of the $H p(\mathrm{UreEG})_{2}(\mathbf{A})$ and the $B p(\mathrm{UreEG})_{2}(\mathbf{C})$ structures. Ribbons are colored as in Figure 2. Residues found at the interface of the complex and known to be involved in metal binding (HpUreE H102, SpUreE H100 and UreG CPH motif residues), as well as the positions of the GTP $\gamma \mathrm{S}$ and GDP molecules (bound to $H p \mathrm{UreG}$ and to $S p \mathrm{UreG}$, respectively), are shown as ball and stick models and the atoms are colored accordingly to the atom type; (B,D) Solvent excluded surfaces of two components of the $H p(\mathrm{UreEG})_{2}(\mathbf{B})$ and the $B p(\mathrm{UreEG})_{2}$ (D) complexes orientated in order to expose the interaction surfaces. The surface is colored according to the surface electrostatic potential (blue, positive; red, negative); (E) Superposition of the structures and of the inertia ellipsoid of $H p(\mathrm{UreEG})_{2}$ (in orange) on the $B p(\mathrm{UreEG})_{2}$ (in blue) model complex. Left and right panels are rotated by $90^{\circ}$ along the horizontal axis, keeping the UreG model as a reference; (F) Ribbon diagram and solvent excluded surface of the best $H p U$ reDFGE docked complex. Ribbons are colored as in Figure 2. Left and right panels are rotated by $90^{\circ}$ along the horizontal axis. 
In 2014, new data provided by NMR allowed a deeper characterization of the $S p$ UreE residues in contact with $\mathrm{S} p \mathrm{UreG}$ [61]. These new findings, in general agreement with the proposed region of $H p \mathrm{UreE}$ interacting with $H p \mathrm{UreG}$ in the docking calculations performed earlier on the same proteins from $H$. pylori [41], prompted the calculation of the $S p$ UreEG complex driven by the NMR experimental information. The calculation used the latest published structure of $S p U r e E$ (PDB id: 4L3K [51]) as well as a model of $S p U r e G$ calculated based on its high degree of homology (sequence identity $=58 \%$ ) with $H p \mathrm{UreG}$, whose crystal structure in complex with $H p \mathrm{UreF}$ and $H p \mathrm{UreD}$ had been released in 2013 [44]. In order to take into account the flexibility of the $S p U$ reE domains, the five low-frequency normal conformational modes of the dimeric $S p U$ reE crystal structure were calculated using the el Némo web-server [62] and a recently published protocol (see ref. [63] and below). The eleven protein conformations determined using this analysis, which comprised the starting structure as well as the ten structures derived by applying the perturbations consistent with each calculated normal mode to the starting structure, were utilized to build a library of structures to be used in the subsequent docking stages. The $S p U$ reE dimeric structure was docked onto the $S p U r e G$ dimeric model complex using the data-driven docking program Haddock 2.1 [22,23] (see above for a general description of the program). For $S p U r e E$, the active residues were those identified by NMR chemical shift and signal intensity perturbations. For $S p U r e G$, the residues in the conserved CPH motif [34] and the most conserved residues on the same side of the protein determined using the server ConSurf [64-66] were used to guide the docking. The general features of the calculated $S p(\mathrm{UreEG})_{2}$ complex (Figure 3C) [61] are similar to those observed in the previously reported complex for $H p(\mathrm{UreEG})_{2}$ [41]. The $S p \mathrm{UreE}$ residues in direct contact with $S p \mathrm{UreG}$ are in good agreement with the experimental data, witnessing the correct outcome of the docking simulation [61]. The $S p U r e E$ surface in the metal binding region is mainly hydrophobic, with some spots in correspondence of positively charged residues. The electrostatic potential mapped on the protein surface of $S p U r e G$ shows that the interaction region is mainly negatively charged (Figure 3D). Therefore, the presence of a divalent cation bound to the $S p U$ reE metal binding site could efficiently change the electrostatic properties of the protein surface, allowing a more favorable interaction with the negatively charged surface of $S p U r e G$ [61], consistently with the stabilization of the UreEG complex in H. pylori upon metal binding [41]. The main difference between the model complexes from S. pasteurii and H. pylori is an anticlockwise rotation of the $S p U$ reE dimer around the vertical axis of about 35 degrees (Figure 3E) [61].

\subsection{Calculation of the Structure of the UreDFGE Complex}

The calculation of the model of the HpUreGE complex released in 2009 (see above) [41], together with the publication of the $H p$ UreDF crystal structure in 2011 (PDB id: 3SF5 [43]), provided the potential to investigate the structural propertied of the putative UreDFGE supercomplex using macromolecular docking, because all the pieces of the puzzle were finally available. A bioinformatics analysis conducted by combining the results obtained from ConSurf [64-66] and ProBis [67-69], together with experimental data from the mutagenesis studies conducted on UreG from H. pylori [43] and K. aerogenes [70], allowed us to identify a restricted set of residues not already involved in other protein-protein interactions on the surfaces of $H p \mathrm{UreF}$ and $H p U r e G$. ConSurf calculates surface residue conservation, while ProBiS predicts surface regions containing structural similarity with known protein binding sites based on 
geometric and physicochemical parameters. Therefore, the HpUreGE complex was docked onto the $H p$ UreDF complex using the docking program Haddock and a protocol identical to that utilized for the calculation of the SpUreGE complex (see above) [61]. The structural models resulting from this docking process were clustered on the basis of their RMSD, allowing the identification of the two most populated clusters (composed of 58 and 51 models, respectively) at a similar Haddock score. The two clusters are indeed representative of the same $H p$ UreDF- $H p$ UreGE model complex because they differ only by the rotation of the symmetric $H p(\mathrm{UreG})_{2}$ homodimer by approximately 180 degrees along a vertical axis perpendicular to the surface of $H p$ UreF. In the resulting modeled structure of the HpUreDFGE complex (Figure 3F), UreG is in direct contact with UreF through weak van der Waals interactions and a number of polar interactions. The shallow crevice formed at the interface between the two UreF monomers is in close contact with the convex surface of the UreG dimer (Figure 3F). Moreover, structural details in the UreF-UreG interaction surface supported the proposition of a role for UreF as a GTPase activating protein (GAP) [36]. Subsequent to the publication of the paper describing the model of the HpUreDFGE complex [71], the crystal structure of the HpUreDFG complex was released (PDB id: 4HI0, [44]). This structure showed that the general secondary and quaternary structure of $H p U r e G$ agrees well with the model structure previously calculated [47], and that the binding site of $H p \mathrm{UreG}$ onto the $H p \mathrm{UreDF}$ complex surface is the same as the one identified earlier [71]. The crystal structure of the complex does not include $H p$ UreE. Unexpectedly, the main difference between the calculated model of the $H p$ UreDFGE complex and the crystal structure of the $H p \mathrm{UreDFG}$ complex is the orientation of the $H p \mathrm{UreG}$ protein dimer with respect to the $H p$ UreF interacting protein: $H p U r e G$ interacts with $H p U r e F$ using the surface patch that was predicted to interact with $H p \mathrm{UreE}$ on the basis of experimental data [41]. In order to explain this difference, it has been proposed that $H p \mathrm{UreG}$, being an intrinsically partially-disordered protein, undergoes the known moonlighting behavior [72], changing its partner as needed along a metabolic pathway $[56,71]$.

\subsection{Calculation of the Structure of the Urease-UreDFG Complex}

In 2012, Ligabue-Braun et al., attempted to build a model of the interaction between $K$. aerogenes urease (KAU) and three of the required accessory proteins $K a \mathrm{UreD}, \mathrm{KaU} \mathrm{UreF}$, and $\mathrm{KaU} \mathrm{UreG}$, in their monomeric oligomerization state [73]. KAU (as well as $S$. pasteurii urease) is a trimer of trimers formed by the three structural urease proteins: UreA, UreB, and UreC [28,29]. The adopted strategy included (i) the homology modelling of the structure of $K a \mathrm{UreG}$ based on the structure of $M j \mathrm{HypB}$ (the structure of $H p$ UreG was not available at the time of the publication) and the homology modelling of the monomeric $\mathrm{KaUreD}$ and $\mathrm{KaUreF}$ based on the structure of $\mathrm{Hp}(\mathrm{UreDF})_{2}$ structure (PDB id: 3SF5, [43]); and (ii) a stepwise docking procedure in which the monomers of $K a U r e D, K a U r e F$, and finally $K a U$ reG were docked on the crystal structure of KAU [73]. All the docking steps, with the exception of the last stage (binding of $\mathrm{KaUreG}$ ), were performed without imposing any restraints. The authors used three different rigid-body docking programs: PatchDock [74], Hex [75], and PIPER [76] via ClusPro 2.0 [77]. PatchDock divides the surface of the two interacting biomolecules into patches according to the surface shape. The obtained patches correspond to patterns that visually distinguish between puzzle pieces. Once the patches are identified, they can be superimposed using shape-matching algorithms that go through three major stages: (i) molecular shape representation; (ii) surface patch matching; and (iii) filtering and scoring. 
Both Hex and PIPER use a FFT method [78] to perform a rigid-body search of the proteins' orientations. Hex accelerates the search by using an expansion of the molecular surface and electric field in spherical harmonics. Fourier correlations between the expansion coefficients are used to simplify the problem of calculating the complementarity between the surfaces in different orientations to that of look up in a table of pre-calculated overlap integrals [79]. PIPER approximates the interaction matrix by its eigenvectors corresponding to the few dominant eigenvalues, resulting in an energy expression written as the sum of a few correlation functions, and by using repeated FFT calculations [76]. In all cases, the results were subsequently clustered with MMTSB Tool Set [80] by hierarchical clustering based on mutual RMSD and evaluated in terms of relative energy with FoldX [81]. The obtained complexes (Figure 4) were checked against experimental small-angle X-ray scattering (SAXS) profiles obtained for the $K$. aerogenes KAU-UreDFG supercomplex [73] as well as against results derived from cross-linking, mutagenesis, and pull-down experiments. Theoretical SAXS profiles were calculated using the FoXS server [82] and compared to the experimental data for the KAU-KaUreD and KAU-KaUreDF complexes [83].

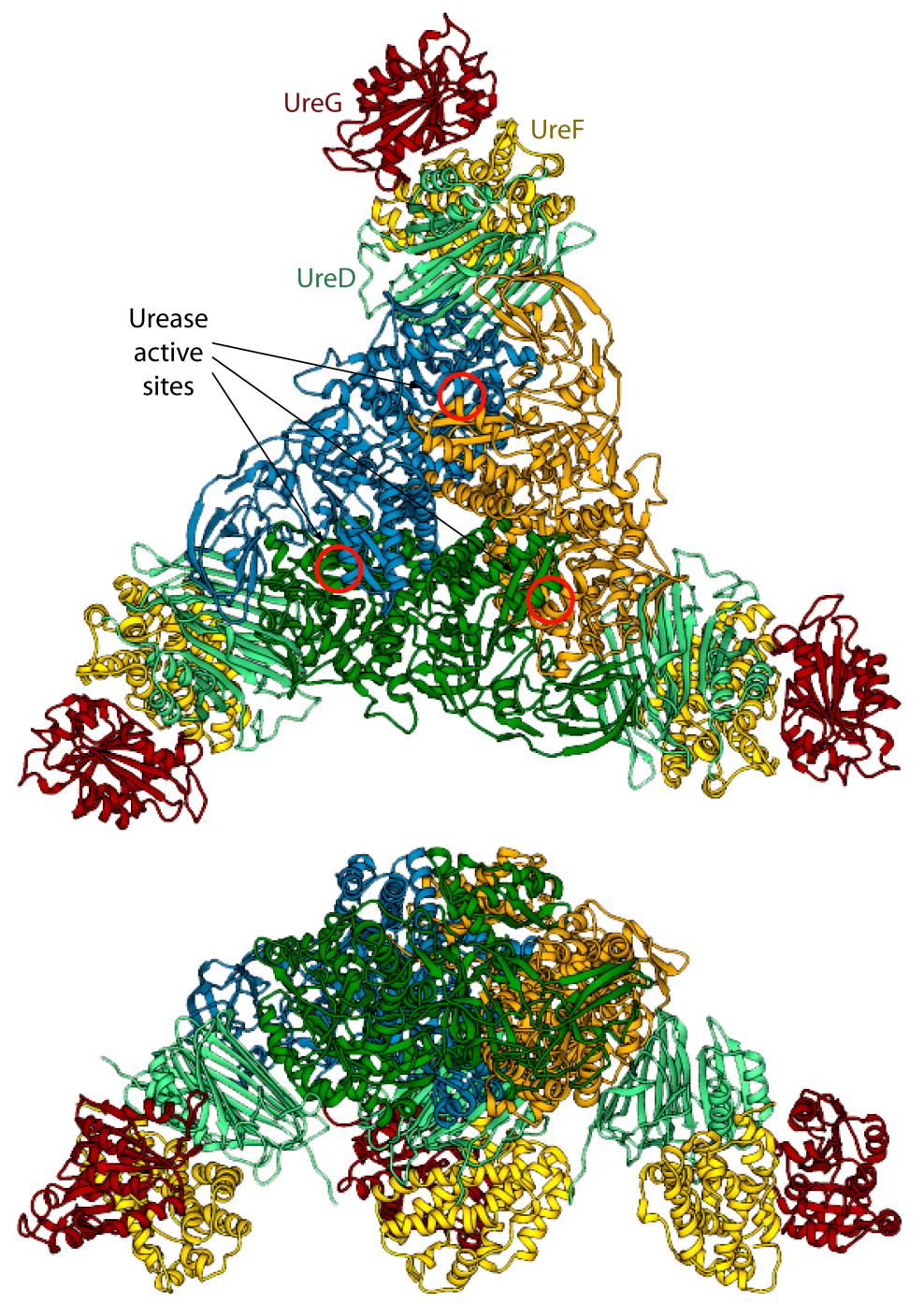

Figure 4. Ribbon scheme of the KAU-UreDFG model complex as calculated in ref. [73]. Ureases subunits and accessory protein are colored as in Figures 1 and 2, respectively. The position of the KAU active sites is indicated by red circles. The views in the top and bottom panel are rotated by $90^{\circ}$ along the horizontal axis. 
The model of the supercomplex is in agreement with pull-down [46] and cross-linking [32] assays. On the other hand, the hinge region of $\mathrm{KaUreB}$ (residues 1-19), which is essential for proper urease activation [84], does not bind directly to any accessory protein. The authors suggested that this region could be required for a proper "gating" of the active site for activation [73]. Furthermore, the accessory proteins bind far away from the urease active site, and a clear pathway for nickel traffic towards the active site was not identified [73]. Finally, the spatial assembly of the urease-UreDFG supercomplex observed in the case of $K$. aerogenes could not be a general case. Indeed, ureases from $H$. pylori or jack bean assume different quaternary structures (the former is a tetramer of trimers of dimers, while the latter is a dimer of trimers of monomers, see Figure 1A) and in such arrangements there is no room for the accessory proteins in the same position of the complex proposed by Ligabue-Braun et al. [73]. The authors suggest that $H$. pylori represents an exception among bacterial ureases, with such difference reflected in a different structural organization of its activation complex, while for plant ureases they hypothesize that the trimer of monomers is activated before the formation of the final protein structure.

\section{HpFur-DNA and HpNikR-DNA Complexes}

Fur and NikR are two important metal-responsive regulators involved in iron and nickel homeostasis and function by controlling gene expression in $H$. pylori. Fur and NikR can bind independently at distinct operators, and also compete for overlapping operators in some co-regulated gene promoters, suggesting a link between iron and nickel regulation [85].

\subsection{HpFur-DNA Complexes}

Iron holds a central position in the host-pathogen interplay in mammals and plants [86]. For instance, iron deficiency is the most common nutritional stress in aquatic environments [87]. In order to manage iron limitation, cells have developed a large number of responses that enable maintenance of iron homeostasis through precise changes in gene expression. The latter allow the cells to adapt their physiology to the environment and to prevent nutrient overloads that would be highly poisonous or lead to oxidative stress [88]. The control of iron metabolism and its coupling with regulation of defenses against oxidative stress is carried out by Fur in most prokaryotic organisms. In H. pylori, Fur is able to act as a transcriptional commutator switch that exploits the alternative readout of DNA grooves to mediate opposite output regulation for the same input signal [63]. In particular, when $\mathrm{Fe}(\mathrm{II})$ ions are abundant, gene transcription is repressed by HpFur in an iron-dependent manner, conforming to the classic Fe(II)-Fur (holo-Fur) repression paradigm [89], in which the iron ion acts as corepressor. Accordingly, the iron-repressible Fur targets include genes involved in Fe(II) ions uptake, such as the prototypical frpB1 gene, which needs to be expressed only under iron-starving conditions [90]. On the other hand, when iron is scarce, apo- $H p$ Fur represses transcription of a different set of genes, including the iron-inducible $p f r$ gene, which codes for a ferritin involved in iron storage, thus demanding derepression only under iron-replete conditions [91]. In this case, the Fe(II) cofactor ion acts as an inducer, rather than a corepressor.

The structure of Fur is a homo-dimer built through a head-to-head interaction of the C-termini of the protein. Each monomer is formed by a DNA-binding domain (DBD) at the $N$-terminal and a metal binding domain (MBD, also called dimerization domain) at the $C$-terminal (Figure 5A). Each Fur monomer binds metal ions in three sites: a regulatory site that can bind one Fe(II) (site S1), a structural site binding one 
$\mathrm{Zn}$ (II) (S2), and a not fully conserved site with uncertain function (S3) (Figure 5A) [92]. Size exclusion chromatography indicated that $H p F u r$ is a dimer in solution and that it is able to tetramerize, and further multimerize, in the presence of divalent metal ions such as Fe(II) and $\mathrm{Mn}(\mathrm{II})$, even in the absence of DNA [63].

To gain insight in the molecular mechanism responsible for the distinctive recognition of apo- and holo-operators, docking simulations were run using the program Haddock and the crystal structure of holo-HpFur (C78S and C150S mutant, PDB code: 2XIG [93]). In this structure there are three Zn(II) ions bound to each of the metal binding site. Curiously, in the regulatory site the $\mathrm{Zn}$ (II) ion is found in two different coordination geometries: in one chain of the dimer the metal ion is tetra-coordinated (S2t in Figure 5A), while in the second chain the $\mathrm{Zn}$ (II) ion adopts a distorted octahedral geometry (S2o). This discrepancy in the coordination geometry can be due to the presence of $\mathrm{Zn}$ (II) ions instead of the physiological Fe(II) ions in the crystallization milieu. Thus, the first step was to use homology modelling to reconstruct a model of the wild type holo- $H p$ Fur featuring both the regulatory metal binding sites in a tetrahedral coordination geometry [63]. The calculation was performed using the Modeller software [60] and the coordination geometry was induced by introducing appropriate symmetry, bond and angle constraints. The model structure of apo-Fur was obtained by depletion of $\mathrm{Zn}$ (II) ions from the model structure of holo-Fur. In order to take into account the experimentally-observed mobility of the DBDs with respect to the MBD [92,93], two libraries of possible Fur conformations were created by analyzing the low-frequency normal modes of the apo- and holo- protein models by using the elNémo web server [62]. In particular, eleven protein conformations (the starting structure, in addition to the structures derived by applying to the starting structure, the perturbation of every calculated normal mode in the two possible directions) determined using this analysis were utilized to build a library of structures for each $H p$ Fur metalation state to be used in subsequent docking calculations together with the apo- and holo-operator $\left(\mathrm{OPI}_{p f r}\right.$ and $\mathrm{OPI}_{f r p B}$ hereafter, respectively) [63]. A starting model for the unbound DNA operators were generated using the DNA analysis and rebuilding software 3DNA implemented in the 3D-DART server [27]. The docking calculations were carried out by considering all the possible combinations between apo-

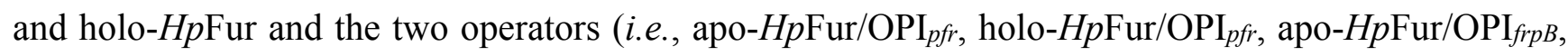
holo- $H p$ Fur/OPI $f r p B$ ). In Fur, $\alpha$-helix 4 is known to be part of the DNA recognition process [94] and in particular the conserved Y65 (Y55 in E. coli numeration) [95] appears to be involved in the binding. Thus, the calculations on the protein side were guided defining as "active" the residue Y65, while the surface residues around it were considered "passive" residues [63]. In the apo- and holo-operators, the base pairs identified in footprinting assay were defined as "active" (bases $-19,-18,-7,-6$ for $\operatorname{OPI}_{p f r}$ and from -25 to -21 and from -19 to -15 for $\mathrm{OPI}_{f r p B}$, see Figure $5 \mathrm{~B}, \mathrm{C}$ ) [63]. In all cases, the docking protocol was optimized by running calculations in the presence and absence of additional restraints on the symmetry of the complex and on the DNA bases [63]. 

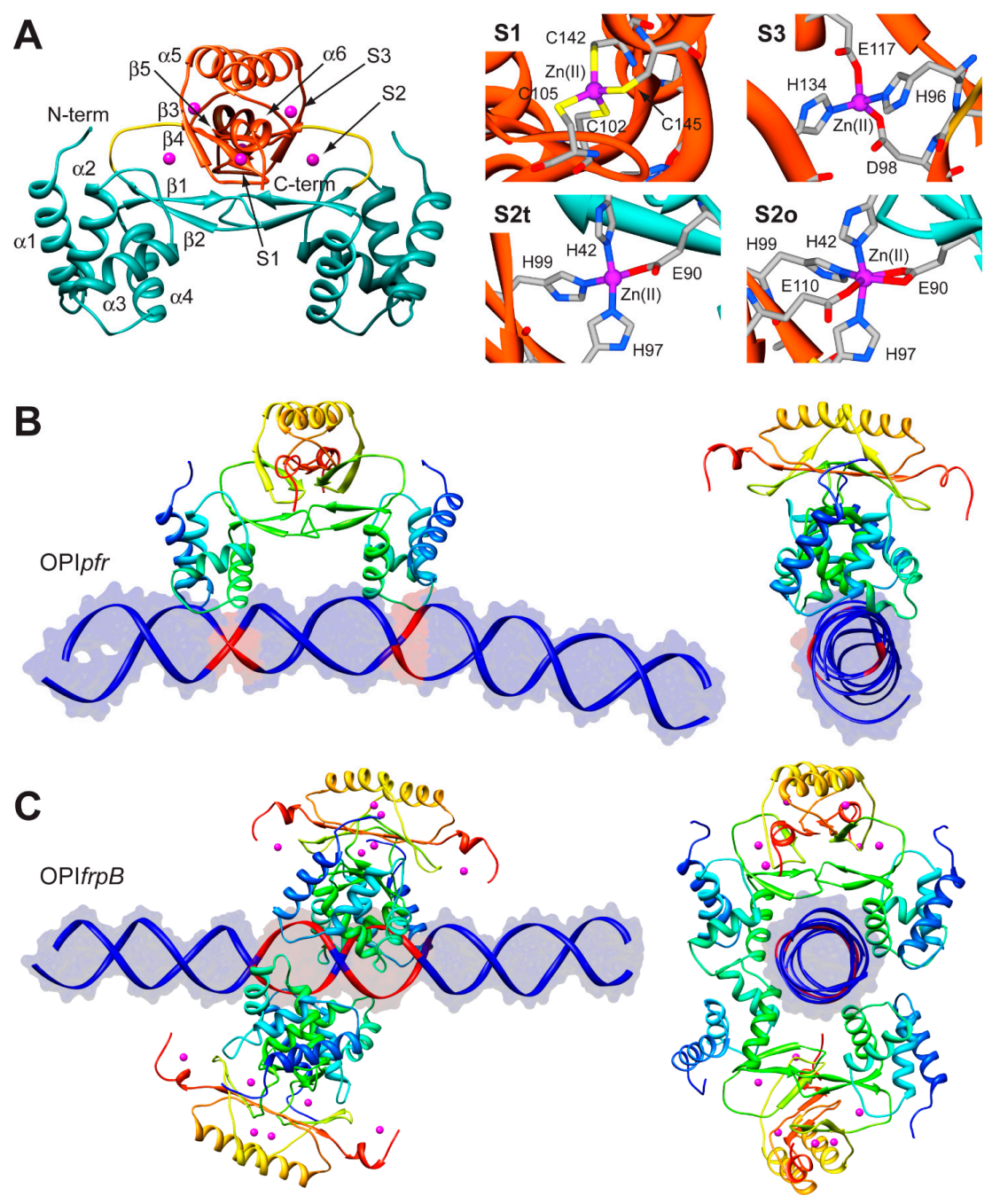

Figure 5. (A) Ribbon scheme and details of the metal binding sites of $H p$ Fur crystal structure. Ribbons for DBD and MBD in left panel are colored in cyan and in orange, respectively, while the unstructured region linking the two domains is in yellow. $\mathrm{Zn}$ (II) ions are in purple. In the right panels, metal binding residues are reported as sticks colored according to atom type; $(\mathbf{B}, \mathbf{C})$ Model structures of the $H p$ Fur-DNA: best docking models resulting for $H p$ Fur-OPI $I_{p f r}(\mathbf{B})$ and Fur-OPI $f r p B(\mathbf{C})$ complexes. The protein is reported as ribbon diagram colored from blue in the proximity of the $N$-terminal to red at the $C$-terminus. $\mathrm{Zn}(\mathrm{II})$ ions are in purple. The DNA is in blue, with the exception of the active residues that are in red.

In the case of $\mathrm{OPI}_{p f r}$ complexes, the best result was obtained for the apo-Fur/OPI $p f r$ model (Figure 5B) with the application of restraints to take into account the symmetry of the complex and the DNA bases known to be involved in the interaction. The calculated protein-DNA complex features the axis connecting the two DNA-binding domains nearly parallel to the DNA major axes. The Fur DNA-binding domains insert the loop between $\alpha$-helices 1 and 2, as well as the first five residues of $\alpha$-helix 4 , in the major groove of the apo-operator in correspondence with the two regions identified by footprinting assay. The apo-operator assumes a convex conformation with respect to the Fur position, broadening the major grooves exposed to the protein and thus promoting protein binding. On the other hand, in simulations carried out on the $\mathrm{OPI}_{f r p B}$ operator, we observed a different orientation of the protein with respect to 
the considered DNA fragment. In all the docking calculations involving OPI ${ }_{f r p B}, H p$ Fur clamps the holo-operator positioning the axis connecting the DNA-binding domains perpendicularly to the DNA major axis. These results, together with the experimental observation of the formation of a complex involving $\mathrm{OPI}_{\text {frp } B}$ and tetrameric holo- $\mathrm{H} p$ Fur, prompted us to perform a simulation in which we included two $H p$ Fur dimers together with the holo-operator. In these simulations we included additional restraints derived from the electrostatic properties of the residues found on the surface of the DNA-binding domain. In particular, our hypothesis was based on the observation that K24 and N58 are placed in good position for inter-protein interaction in the case of the formation of a tetramer build by connecting two Fur dimers across the DNA-binding domain. In the resulting best complex (Figure 5C), the two Fur dimers envelop the DNA using both the DNA-binding domain and the dimerization domain, completely covering the large region of the operator identified by footprinting assay. The Fur tetramer does not bind DNA perpendicularly to the operator major axes, but is tilted by about 30 degrees, allowing both Fur dimers to putatively interact with DNA regions that are feebly detected in the footprinting assay (Figure 5C) [63].

\subsection{HpNikR-DNA Complex}

NikR is a transcription factor that regulates the expression of genes coding for proteins involved in nickel metabolism [28,96]. It is a highly homologous protein, found in $c a .30$ species of bacteria and archea. The Ni(II)-bound NikR from E. coli $(E c N i k R)$ binds to DNA and represses the transcription of the nikABCDE operon, which codes for a specific Ni(II) membrane uptake ABC transporter [97,98]. On the other hand, NikR from H. pylori $(H p N i k R)$ is a pleiotropic regulator of several genes, acting as a nickel-dependent repressor of $H p$ NikR itself and of the Ni(II) permease NixA, as well as a nickel-dependent activator of urease operon [99,100]. Numerous crystal structures of NikR [101-103] have established that this protein is a homo-tetramer, made of a dimer of dimers, constituted by two domains (Figure 6A). One domain is the central metal binding domain (MBD), made of the $C$-terminal portion of the protein responsible for tetramerization. This domain hosts four regulatory metal binding sites symmetrically located at the tetramerization interface, where $\mathrm{Ni}$ (II) ions bind three fully conserved histidines and one cysteine residues in a square planar coordination geometry (HHHC site hereafter, Figure 6A). Some crystal structures of full-length $H p N i k R$ [101,103], showing partial occupancy of the square planar HHHC sites as well as additional $\mathrm{Ni}$ (II) binding sites, have been interpreted as indicating means for $H p N i k R$ to recognize different DNA sequences depending on the level of $\mathrm{Ni}$ (II) concentration and cytoplasmic $\mathrm{pH}$, but no evidence has been obtained so far to support this hypothesis-The MBD is flanked by two peripheral DNA binding domains (DBD), separated by flexible linkers. Each DBD is made of the dimerization of the $N$-terminal portion of the protein and features a ribbon-helix-helix motif for DNA binding, typical of prokaryotic transcription factors [104], in which two anti-parallel $N$-terminal $\beta$-strands from opposite protomers make a two-stranded anti-parallel $\beta$-sheet that contacts the major groove (Figure 6A). Three distinct conformations of NikR have been observed in the solid-state, characterized by the position of the DBDs with respect to the central MBD, depending on the conformation of the flexible link between the domains: cis, open, and trans (Figure 6B). The crystal structure of the EcNikR-DNA complex has shown that the cis conformation of the Ni(II)-bound protein is able to bind DNA with the MBD that keeps DBDs at the right distance to contact one-half-site of a two-fold symmetric DNA operator 
(Figure 6A) [105]. The $\mathrm{Ni}(\mathrm{II})$ binding to the $\mathrm{MBD}$ of $H p$ NikR results in an increase of the protein affinity to DNA, as determined by calorimetric titrations [106]. The way through which Ni(II) binding propagates the information for DNA binding at the MBD along the protein chain to the DBDs, has been investigated both experimentally $[100,107,108]$, and computationally, using atomistic molecular dynamics simulations [100,109,110]. The conclusions suggest the occurrence of an ensemble of interconverting structures in solution, spanning the open, trans and cis conformers, both for the apo and the holo-protein. This implies that $\mathrm{Ni}$ (II) binding does not induce a conformational rearrangement of the protein towards a specific cis conformation able to bind DNA, but rather unlocks the movement of the two peripheral $N$-terminal DNA-binding domains with respect to the central $C$-terminal metal binding domain $[100,110]$.

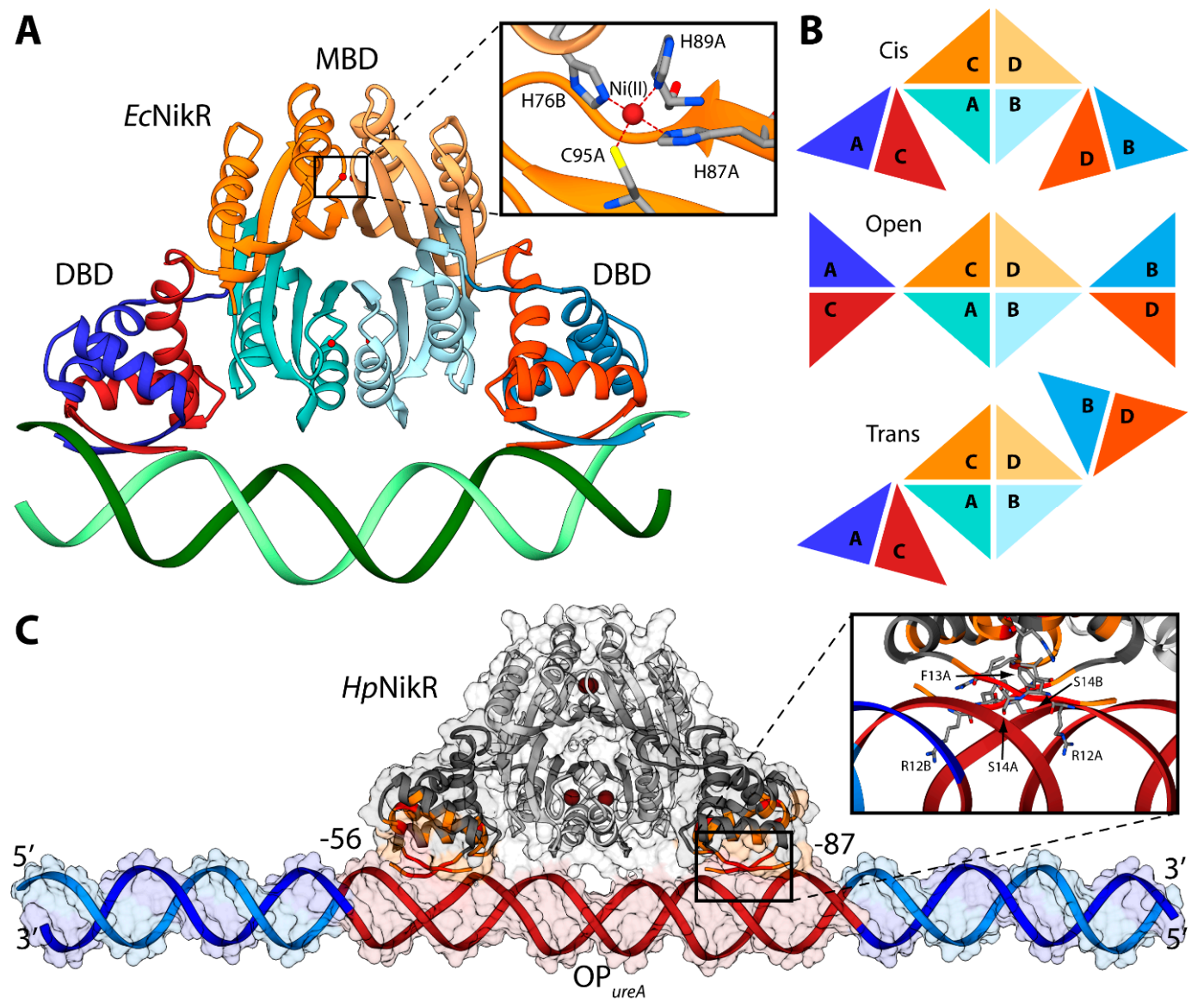

Figure 6. (A) Ribbon scheme of the crystal structure of holo- $E c$ NikR in complex with DNA. DBD are in orange and blue, while MBD are in light orange and light orange. Nickel ions are reported as dark red spheres and DNA is in green. The inset reports the details of the $\mathrm{Ni}$ (II) binding site. Nickel binding residues are reported as sticks colored according to atom type; (B) Schematic representation of the conformations of NikR found in the available crystal structures; (C) Ribbon diagram and solvent-excluded surface of the model structure of the $H p$ NikR-DNA complex. $H p$ NikR ribbons are colored in dark and light grey in the $\mathrm{DBD}$ and MBD, respectively. Active and passive residues used to guide the docking are in red and orange, respectively. $\mathrm{Ni}(\mathrm{II})$ ions are reported as dark brown spheres. The DNA ribbons are in dark red for the $H p N i k R$ operator and in light and dark blue for the terminal regions. The inset shows the details of $H p$ NikR residues in the DBD in direct contact with the DNA major groove. 
Some molecular details of the $H p N i k R$ interaction with the urease operator $\mathrm{OP}_{u r e A}$, based primarily on NMR spectroscopy in solution, have recently been reported [111]. Considering that the size of the full-length protein prevents the characterization of the $H p$ NikR-OP ure interaction using only NMR, the two protein domains were investigated separately. An NMR-based analysis of the perturbations, induced on the DBD by the presence of $\mathrm{OP}_{\text {urea, }}$, provided information useful to guide the subsequent docking

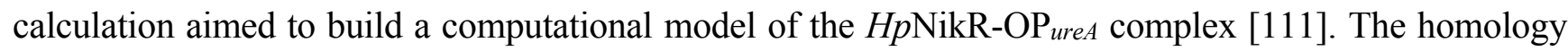
model of the holo- $H p$ NikR in the cis conformation was calculated in a previous study [100]. The modeling was based on the structure of NikR from E. coli in the cis conformation (PDB ID: 2HZV, resolution $3.00 \AA)$ [105]. The alignment between the sequences of NikR from all the available crystal structures (H. pylori, E. coli, and P. horikoshii) was produced using ClustalW [112] and manually adjusted in order to match the primary and secondary structure of the proteins. The obtained alignment was used to calculate 50 structural models of the cis conformation of tetrameric $H p$ NikR using the program Modeller [60]. Four square-planar Ni(II) ions were included in the modeling, bound in the well-known metal binding sites involving H88 from one monomer and H99, H101 and C107 from the adjacent monomer [100,111]. A starting model for the unbound $\mathrm{OP}_{\text {ureA }}$ operator was generated using the DNA analysis and rebuilding software 3DNA implemented in the 3D-DART server [27]. OP ${ }_{\text {ure }}$ comprises nucleotides from -56 to -87 with respect to the urease operon transcriptional start site in H. pylori strain G27 [111]. In order to avoid biasing effects due to the highly charged DNA termini, twenty nucleotides were added to each side of the operator by using the $H$. pylori strain G27 genome. The model was generated in the canonical B-DNA conformation. The holo- $H p$ NikR model structure in the cis conformation was docked onto $\mathrm{OP}_{\text {ure }}$ using the data-driven docking program HADDOCK 2.1 and the same protocol used previously in the case of Fur (see ref. [63] and above). The calculation was guided by selecting the protein residues found by NMR to be involved in the interaction with DNA (R12, F13, S14, V15, S16, S36, R37, L40), as well as the operator's nucleotides (from -56 to -87 with respect to the urease operon transcriptional start site in H. pylori strain G27).

The NMR-based docking model of the interaction complex between the full-length $H p$ NikR and $\mathrm{OP}_{\text {ure }}$ (Figure 6C), obtained using the NMR-based constraints described above, has strong analogies with the crystal structure of the analogous complex experimentally determined in E. coli [105]. The major axes of $H p N i k R$ and $\mathrm{OP}_{\text {ure }}$ are almost parallel, with the latter uninterruptedly making contacts with $H p N i k R$, in agreement with DNAase I footprinting assays [106]. The DNA major axis is slightly bent, even though not as much as in the E. coli structure, while the major groove is more open in the region of interaction with the DBD domain, causing in turn shrinkage of the minor groove in the same region (Figure $6 \mathrm{C}$ ). $H p$ NikR appears to interact with DNA mainly by inserting residues $12-14$ of the DBD into the major groove in the proximity of nucleotides positioned from -24 to -26 and from -80 to -82 with respect to the urease operon transcriptional start site in H. pylori strain G27 (Figure 6C) [111].

\section{Perspectives}

The determination of the structure of the complexes between proteins, as well as between proteins and gene transcription operators, is an important step not only in the advancement of the basic knowledge of a metabolic process, but also in the identification of new targets for the development of new drugs. 
Moreover, several diseases are caused by alterations of transcription and/or protein-DNA interactions, making these mechanisms highly attractive targets for therapy [113].

Current methods and libraries for drug discovery work well with a limited number of targets, such as enzymes, ion channels or receptors, which feature a well-defined and structurally-stable concave binding sites. The G protein-coupled receptors (GPCRs), protein kinases and proteases super-families are good examples of this kind of targets with "druggable" characteristics [114-118]. Targeting protein-protein interfaces (PPIs) of multi-protein complexes that mediate cell regulation (long regarded by many as "undruggable") has become a subject of intense activity in recent years [119]. Within protein-protein complexes of known structure, PPIs are generally large (ca. 1500-3000 $\AA^{2}$ ), flat, and relatively featureless [120], making the design of small molecule antagonists a challenging task. Furthermore, in the case of protein-DNA interactions, many factors have led to consider transcription as"undruggable" [121]. Indeed, several DNA binding proteins lack ligand-binding domains or intrinsic enzymatic activities [121]. Moreover, some of the protein domains involved in DNA recognition or protein recognition are intrinsically disordered in the absence of interacting partners [122,123]. Finally, the complexity of the interactions involving multi-point contacts over large surfaces, the lack of defined pockets suitable for a rational design of small molecules, and the presence of metal ions are critical issues that need to be addressed [121].

The development of protocols to mimic these difficult interactions is a key answer to these pressing needs, with the potential to offer the molecular details of new druggable targets. First it has been recognized that energetic hotspots contribute much of interface interaction free energy in many PPIs [124], but also it has been noted that many interactions involve continuous epitopes constituting defined grooves or series of small specific pockets [125]. Such observations lead to the development of stapled $\alpha$-helical peptides and other proteo-mimetic approaches to drugging interfaces [126]. In a different approach, it has been noted that fragments might gain low-affinity "footholds" on PPIs, and that these might be elaborated to apt modulators of multi-protein assemblies where knowledge of the structures of the complexes is available [114]. In the latter examples, the experimental determination or the computational prediction of the structural details of the protein-protein or the protein-DNA interface is mandatory to the study of the interaction interfaces. In this aim, the macromolecular docking protocols discussed in this review have evolved in the last twenty years thanks to the development of new algorithms and the availability of more computational power, and have become an important complement to the experimental techniques.

\section{Acknowledgements}

FM was supported by the University of Bologna through the FARB Program (Finanziamenti dell'Alma Mater Studiorum alla Ricerca di Base) "Modulation of protein-DNA interactions with small molecules: novel opportunities for drug design”.

\section{Conflicts of Interest}

The authors declare no conflict of interest. 


\section{References}

1. Cozzarelli, N.R.; Cost, G.J.; Nollmann, M.; Viard, T.; Stray, J.E. Giant proteins that move DNA: Bullies of the genomic playground. Nat. Rev. Mol. Cell Biol. 2006, 7, 580-588.

2. Wodak, S.J.; Vlasblom, J.; Turinsky, A.L.; Pu, S. Protein-protein interaction networks: The puzzling riches. Curr. Opin. Struct. Biol. 2013, 23, 941-953.

3. Lage, K. Protein-protein interactions and genetic diseases: The interactome. Biochim. Biophys. Acta 2014, 1842, 1971-1980.

4. Re, A.; Joshi, T.; Kulberkyte, E.; Morris, Q.; Workman, C.T. RNA-protein interactions: An overview. Methods Mol. Biol. 2014, 1097, 491-521.

5. Su, C.; Peregrin-Alvarez, J.M.; Butland, G.; Phanse, S.; Fong, V.; Emili, A.; Parkinson, J. Bacteriome.org-An integrated protein interaction database for E. coli. Nucleic Acids Res. 2008, 36, D632-D636.

6. Braun, P.; Aubourg, S.; van Leene, J.; de Jaeger, G.; Lurin, C. Plant protein interactomes. Annu. Rev. Plant Biol. 2013, 64, 161-187.

7. Venkatesan, K.; Rual, J.F.; Vazquez, A.; Stelzl, U.; Lemmens, I.; Hirozane-Kishikawa, T.; Hao, T.; Zenkner, M.; Xin, X.; Goh, K.I.; et al. An empirical framework for binary interactome mapping. Nat. Methods 2009, 6, 83-90.

8. Stumpf, M.P.; Thorne, T.; de Silva, E.; Stewart, R.; An, H.J.; Lappe, M.; Wiuf, C. Estimating the size of the human interactome. Proc. Natl. Acad. Sci. USA 2008, 105, 6959-6964.

9. Cukuroglu, E.; Gursoy, A.; Nussinov, R.; Keskin, O. Non-redundant unique interface structures as templates for modeling protein interactions. PLOS ONE 2014, 9, e86738.

10. Rodrigues, J.P.; Bonvin, A.M. Integrative computational modeling of protein interactions. FEBS J. 2014, 281, 1988-2003.

11. Russell, R.B.; Alber, F.; Aloy, P.; Davis, F.P.; Korkin, D.; Pichaud, M.; Topf, M.; Sali, A. A structural perspective on protein-protein interactions. Curr. Opin. Struct. Biol. 2004, 14, 313-324.

12. Moreira, I.S.; Fernandes, P.A.; Ramos, M.J. Protein-protein docking dealing with the unknown. J. Comput. Chem. 2010, 31, 317-342.

13. Grosdidier, S.; Fernandez-Recio, J. Protein-protein docking and hot-spot prediction for drug discovery. Curr. Pharm. Des. 2012, 18, 4607-4618.

14. Huang, S.Y. Search strategies and evaluation in protein-protein docking: Principles, advances and challenges. Drug Discov. Today 2014, 19, 1081-1096.

15. Huang, S.Y. Exploring the potential of global protein-protein docking: An overview and critical assessment of current programs for automatic ab initio docking. Drug Discov. Today 2015.

16. Van Dijk, M.; Bonvin, A.M. A protein-DNA docking benchmark. Nucleic Acids Res. 2008, 36, e88.

17. Van Dijk, M.; Visscher, K.M.; Kastritis, P.L.; Bonvin, A.M. Solvated protein-DNA docking using HADDOCK. J. Biomol. NMR 2013, 56, 51-63.

18. Tuszynska, I.; Matelska, D.; Magnus, M.; Chojnowski, G.; Kasprzak, J.M.; Kozlowski, L.P.; Dunin-Horkawicz, S.; Bujnicki, J.M. Computational modeling of protein-RNA complex structures. Methods 2014, 65, 310-319. 
19. Huang, S.Y.; Grinter, S.Z.; Zou, X. Scoring functions and their evaluation methods for protein-ligand docking: Recent advances and future directions. Phys. Chem. Chem. Phys. 2010, 12, 12899-12908.

20. Muegge, I. PMF scoring revisited. J. Med. Chem. 2006, 49, 5895-5902.

21. Van Dijk, M.; van Dijk, A.D.; Hsu, V.; Boelens, R.; Bonvin, A.M. Information-driven protein-DNA docking using HADDOCK: It is a matter of flexibility. Nucleic Acids Res. 2006, 34, 3317-3325.

22. Dominguez, C.; Boelens, R.; Bonvin, A.M. HADDOCK: A protein-protein docking approach based on biochemical or biophysical information. J. Am. Chem. Soc. 2003, 125, 1731-1737.

23. De Vries, S.J.; van Dijk, A.D.; Krzeminski, M.; van Dijk, M.; Thureau, A.; Hsu, V.; Wassenaar, T.; Bonvin, A.M. HADDOCK versus HADDOCK: New features and performance of HADDOCK2.0 on the CAPRI targets. Proteins 2007, 69, 726-733.

24. Van Dijk, M.; Bonvin, A.M. Pushing the limits of what is achievable in protein-DNA docking: Benchmarking HADDOCK's performance. Nucleic Acids Res. 2010, 38, 5634-5647.

25. Brunger, A.T.; Adams, P.D.; Clore, G.M.; DeLano, W.L.; Gros, P.; Grosse-Kunstleve, R.W.; Jiang, J.S.; Kuszewski, J.; Nilges, M.; Pannu, N.S.; et al. Crystallography \& NMR system: A new software suite for macromolecular structure determination. Acta Crystallogr. D Biol. Crystallogr. 1998, 54, 905-921.

26. Linge, J.P.; O’Donoghue, S.I.; Nilges, M. Automated assignment of ambiguous nuclear overhauser effects with ARIA. Methods Enzymol. 2001, 339, 71-90.

27. Van Dijk, M.; Bonvin, A.M. 3D-DART: A DNA structure modelling server. Nucleic Acids Res. 2009, 37, W235-W239.

28. Zambelli, B.; Musiani, F.; Benini, S.; Ciurli, S. Chemistry of $\mathrm{Ni}^{2+}$ in urease: Sensing, trafficking, and catalysis. Acc. Chem. Res. 2011, 44, 520-530.

29. Maroney, M.J.; Ciurli, S. Nonredox nickel enzymes. Chem. Rev. 2014, 114, 4206-4228.

30. Farrugia, M.A.; Macomber, L.; Hausinger, R.P. Biosynthesis of the urease metallocenter. J. Biol. Chem. 2013, 288, 13178-13185.

31. Steyert, S.R.; Rasko, D.A.; Kaper, J.B. Functional and phylogenetic analysis of ureD in Shiga toxin-producing Escherichia coli. J. Bacteriol. 2011, 193, 875-886.

32. Chang, Z.; Kuchar, J.; Hausinger, R.P. Chemical cross-linking and mass spectrometric identification of sites of interaction for UreD, UreF, and urease. J. Biol. Chem. 2004, 279, 15305-15313.

33. Soriano, A.; Hausinger, R.P. GTP-dependent activation of urease apoprotein in complex with the UreD, UreF, and UreG accessory proteins. Proc. Natl. Acad. Sci. USA 1999, 96, 11140-11144.

34. Zambelli, B.; Stola, M.; Musiani, F.; de Vriendt, K.; Samyn, B.; Devreese, B.; van Beeumen, J.; Turano, P.; Dikiy, A.; Bryant, D.A.; et al. UreG, a chaperone in the urease assembly process, is an intrinsically unstructured GTPase that specifically binds $\mathrm{Zn}^{2+}$. J. Biol. Chem. 2005, 280, 4684-4695.

35. Musiani, F.; Ippoliti, E.; Micheletti, C.; Carloni, P.; Ciurli, S. Conformational fluctuations of UreG, an intrinsically disordered enzyme. Biochemistry 2013, 52, 2949-2954.

36. Salomone-Stagni, M.; Zambelli, B.; Musiani, F.; Ciurli, S. A model-based proposal for the role of UreF as a GTPase-activating protein in the urease active site biosynthesis. Proteins 2007, 68, $749-761$. 
37. Carter, E.L.; Flugga, N.; Boer, J.L.; Mulrooney, S.B.; Hausinger, R.P. Interplay of metal ions and urease. Metallomics 2009, 1, 207-221.

38. Soriano, A.; Colpas, G.J.; Hausinger, R.P. UreE stimulation of GTP-dependent urease activation in the UreD-UreF-UreG-urease apoprotein complex. Biochemistry 2000, 39, 12435-12440.

39. Rain, J.C.; Selig, L.; de Reuse, H.; Battaglia, V.; Reverdy, C.; Simon, S.; Lenzen, G.; Petel, F.; Wojcik, J.; Schachter, V.; et al. The protein-protein interaction map of Helicobacter pylori. Nature 2001, 409, 211-215.

40. Voland, P.; Weeks, D.L.; Marcus, E.A.; Prinz, C.; Sachs, G.; Scott, D. Interactions among the seven Helicobacter pylori proteins encoded by the urease gene cluster. Am. J. Physiol. Gastrointest. Liver Physiol. 2003, 284, G96-G106.

41. Bellucci, M.; Zambelli, B.; Musiani, F.; Turano, P.; Ciurli, S. Helicobacter pylori UreE, a urease accessory protein: Specific $\mathrm{Ni}(2+)$ - and $\mathrm{Zn}(2+)$-binding properties and interaction with its cognate UreG. Biochem. J. 2009, 422, 91-100.

42. Lam, R.; Romanov, V.; Johns, K.; Battaile, K.P.; Wu-Brown, J.; Guthrie, J.L.; Hausinger, R.P.; Pai, E.F.; Chirgadze, N.Y. Crystal structure of a truncated urease accessory protein UreF from Helicobacter pylori. Proteins 2010, 78, 2839-2848.

43. Fong, Y.H.; Wong, H.C.; Chuck, C.P.; Chen, Y.W.; Sun, H.; Wong, K.B. Assembly of preactivation complex for urease maturation in Helicobacter pylori: Crystal structure of UreF-UreH protein complex. J. Biol. Chem. 2011, 286, 43241-43249.

44. Fong, Y.H.; Wong, H.C.; Yuen, M.H.; Lau, P.H.; Chen, Y.W.; Wong, K.B. Structure of UreG/UreF/UreH complex reveals how urease accessory proteins facilitate maturation of Helicobacter pylori urease. PLoS Biol. 2013, 11, e1001678.

45. Leipe, D.D.; Wolf, Y.I.; Koonin, E.V.; Aravind, L. Classification and evolution of P-loop GTPases and related ATPases. J. Mol. Biol. 2002, 317, 41-72.

46. Boer, J.L.; Quiroz-Valenzuela, S.; Anderson, K.L.; Hausinger, R.P. Mutagenesis of Klebsiella aerogenes $\mathrm{UreG}$ to probe nickel binding and interactions with other urease-related proteins. Biochemistry 2010, 49, 5859-5869.

47. Zambelli, B.; Turano, P.; Musiani, F.; Neyroz, P.; Ciurli, S. Zn ${ }^{2+}$-linked dimerization of UreG from Helicobacter pylori, a chaperone involved in nickel trafficking and urease activation. Proteins 2009, 74, 222-239.

48. Real-Guerra, R.; Staniscuaski, F.; Zambelli, B.; Musiani, F.; Ciurli, S.; Carlini, C.R. Biochemical and structural studies on native and recombinant Glycine max UreG: A detailed characterization of a plant urease accessory protein. Plant. Mol. Biol. 2012, 78, 461-475.

49. Zambelli, B.; Musiani, F.; Savini, M.; Tucker, P.; Ciurli, S. Biochemical studies on Mycobacterium tuberculosis UreG and comparative modeling reveal structural and functional conservation among the bacterial UreG family. Biochemistry 2007, 46, 3171-3182.

50. Remaut, H.; Safarov, N.; Ciurli, S.; van Beeumen, J. Structural basis for $\mathrm{Ni}^{2+}$ transport and assembly of the urease active site by the metallochaperone UreE from Bacillus pasteurii. J. Biol. Chem. 2001, 276, 49365-49370.

51. Zambelli, B.; Banaszak, K.; Merloni, A.; Kiliszek, A.; Rypniewski, W.; Ciurli, S. Selectivity of $\mathrm{Ni}(\mathrm{II})$ and $\mathrm{Zn}$ (II) binding to Sporosarcina pasteurii UreE, a metallochaperone in the urease assembly: A calorimetric and crystallographic study. J. Biol. Inorg. Chem. 2013, 18, 1005-1017. 
52. Song, H.K.; Mulrooney, S.B.; Huber, R.; Hausinger, R.P. Crystal structure of Klebsiella aerogenes UreE, a nickel-binding metallochaperone for urease activation. J. Biol. Chem. 2001, 276, 49359-49364.

53. Shi, R.; Munger, C.; Asinas, A.; Benoit, S.L.; Miller, E.; Matte, A.; Maier, R.J.; Cygler, M. Crystal structures of apo and metal-bound forms of the UreE protein from Helicobacter pylori: Role of multiple metal binding sites. Biochemistry 2010, 49, 7080-7088.

54. Banaszak, K.; Martin-Diaconescu, V.; Bellucci, M.; Zambelli, B.; Rypniewski, W.; Maroney, M.J.; Ciurli, S. Crystallographic and X-ray absorption spectroscopic characterization of Helicobacter pylori UreE bound to $\mathrm{Ni}^{2+}$ and $\mathrm{Zn}^{2+}$ reveals a role for the disordered $C$-terminal arm in metal trafficking. Biochem. J. 2012, 441, 1017-1026.

55. Musiani, F.; Zambelli, B.; Stola, M.; Ciurli, S. Nickel trafficking: Insights into the fold and function of UreE, a urease metallochaperone. J. Inorg. Biochem. 2004, 98, 803-813.

56. Yang, X.; Li, H.; Lai, T.P.; Sun, H. UreE-UreG complex facilitates nickel transfer and preactivates GTPase of UreG in Helicobacter pylori. J. Biol. Chem. 2015, 290, 12474-12485.

57. Grossoehme, N.E.; Mulrooney, S.B.; Hausinger, R.P.; Wilcox, D.E. Thermodynamics of $\mathrm{Ni}^{2+}$, $\mathrm{Cu}^{2+}$, and $\mathrm{Zn}^{2+}$ binding to the urease metallochaperone UreE. Biochemistry 2007, 46, 10506-10516.

58. Gasper, R.; Scrima, A.; Wittinghofer, A. Structural insights into HypB, a GTP-binding protein that regulates metal binding. J. Biol. Chem. 2006, 281, 27492-27502.

59. Gray, J.J.; Moughon, S.; Wang, C.; Schueler-Furman, O.; Kuhlman, B.; Rohl, C.A.; Baker, D. Protein-protein docking with simultaneous optimization of rigid-body displacement and side-chain conformations. J. Mol. Biol. 2003, 331, 281-299.

60. Marti-Renom, M.A.; Stuart, A.C.; Fiser, A.; Sanchez, R.; Melo, F.; Sali, A. Comparative protein structure modeling of genes and genomes. Annu. Rev. Biophys. Biomol. Struct. 2000, 29, 291-325.

61. Merloni, A.; Dobrovolska, O.; Zambelli, B.; Agostini, F.; Bazzani, M.; Musiani, F.; Ciurli, S. Molecular landscape of the interaction between the urease accessory proteins UreE and UreG. Biochim. Biophys. Acta 2014, 1844, 1662-1674.

62. Suhre, K.; Sanejouand, Y.H. ElNemo: A normal mode web server for protein movement analysis and the generation of templates for molecular replacement. Nucleic Acids Res. 2004, 32, W610-W614.

63. Agriesti, F.; Roncarati, D.; Musiani, F.; del Campo, C.; Iurlaro, M.; Sparla, F.; Ciurli, S.; Danielli, A.; Scarlato, V. FeON-FeOFF: The Helicobacter pylori Fur regulator commutates iron-responsive transcription by discriminative readout of opposed DNA grooves. Nucleic Acids Res. 2014, 42, 3138-3151.

64. Glaser, F.; Pupko, T.; Paz, I.; Bell, R.E.; Bechor-Shental, D.; Martz, E.; Ben-Tal, N. ConSurf: Identification of functional regions in proteins by surface-mapping of phylogenetic information. Bioinformatics 2003, 19, 163-164.

65. Landau, M.; Mayrose, I.; Rosenberg, Y.; Glaser, F.; Martz, E.; Pupko, T.; Ben-Tal, N. ConSurf 2005: The projection of evolutionary conservation scores of residues on protein structures. Nucleic Acids Res. 2005, 33, W299-W302.

66. Ashkenazy, H.; Erez, E.; Martz, E.; Pupko, T.; Ben-Tal, N. ConSurf 2010: Calculating evolutionary conservation in sequence and structure of proteins and nucleic acids. Nucleic Acids Res. 2010, 38, W529-W533. 
67. Konc, J.; Janezic, D. ProBiS algorithm for detection of structurally similar protein binding sites by local structural alignment. Bioinformatics 2010, 26, 1160-1168.

68. Konc, J.; Janezic, D. ProBiS: A web server for detection of structurally similar protein binding sites. Nucleic Acids Res. 2010, 38, W436-W440.

69. Konc, J.; Janezic, D. ProBiS-2012: Web server and web services for detection of structurally similar binding sites in proteins. Nucleic Acids Res. 2012, 40, W214-W221.

70. Boer, J.L.; Hausinger, R.P. Klebsiella aerogenes UreF: Identification of the UreG binding site and role in enhancing the fidelity of urease activation. Biochemistry 2012, 51, 2298-2308.

71. Biagi, F.; Musiani, F.; Ciurli, S. Structure of the UreD-UreF-UreG-UreE complex in Helicobacter pylori: A model study. J. Biol. Inorg. Chem. 2013, 18, 571-577.

72. Tompa, P.; Szasz, C.; Buday, L. Structural disorder throws new light on moonlighting. Trends Biochem. Sci. 2005, 30, 484-489.

73. Ligabue-Braun, R.; Real-Guerra, R.; Carlini, C.R.; Verli, H. Evidence-based docking of the urease activation complex. J. Biomol. Struct. Dyn. 2013, 31, 854-861.

74. Schneidman-Duhovny, D.; Inbar, Y.; Nussinov, R.; Wolfson, H.J. PatchDock and SymmDock: Servers for rigid and symmetric docking. Nucleic Acids Res. 2005, 33, W363-W367.

75. Macindoe, G.; Mavridis, L.; Venkatraman, V.; Devignes, M.D.; Ritchie, D.W. HexServer: An FFT-based protein docking server powered by graphics processors. Nucleic Acids Res. 2010, 38, W445-W449.

76. Kozakov, D.; Brenke, R.; Comeau, S.R.; Vajda, S. PIPER: An FFT-based protein docking program with pairwise potentials. Proteins 2006, 65, 392-406.

77. Comeau, S.R.; Gatchell, D.W.; Vajda, S.; Camacho, C.J. ClusPro: A fully automated algorithm for protein-protein docking. Nucleic Acids Res. 2004, 32, W96-W99.

78. Harrison, R.W.; Kourinov, I.V.; Andrews, L.C. The Fourier-Green's function and the rapid evaluation of molecular potentials. Protein. Eng. 1994, 7, 359-369.

79. Ritchie, D.W.; Kemp, G.J.L. Protein docking using spherical polar Fourier correlations. Proteins 2000, 39, 178-194.

80. Feig, M.; Karanicolas, J.; Brooks, C.L., 3rd. MMTSB Tool Set: Enhanced sampling and multiscale modeling methods for applications in structural biology. J. Mol. Graph. Model. 2004, 22, 377-395.

81. Guerois, R.; Nielsen, J.E.; Serrano, L. Predicting Changes in the Stability of Proteins and Protein Complexes: A Study of More Than 1000 Mutations. J. Mol. Biol. 2002, 320, 369-387.

82. Schneidman-Duhovny, D.; Hammel, M.; Sali, A. FoXS: A web server for rapid computation and fitting of SAXS profiles. Nucleic Acids Res. 2010, 38, W540-W544.

83. Quiroz-Valenzuela, S.; Sukuru, S.C.; Hausinger, R.P.; Kuhn, L.A.; Heller, W.T. The structure of urease activation complexes examined by flexibility analysis, mutagenesis, and small-angle X-ray scattering. Arch. Biochem. Biophys. 2008, 480, 51-57.

84. Carter, E.L.; Tronrud, D.E.; Taber, S.R.; Karplus, P.A.; Hausinger, R.P. Iron-containing urease in a pathogenic bacterium. Proc. Natl. Acad. Sci. USA 2011, 108, 13095-13099.

85. Delany, I.; Ieva, R.; Soragni, A.; Hilleringmann, M.; Rappuoli, R.; Scarlato, V. In vitro analysis of protein-operator interactions of the NikR and fur metal-responsive regulators of coregulated genes in Helicobacter pylori. J. Bacteriol. 2005, 187, 7703-7715. 
86. Nairz, M.; Schroll, A.; Sonnweber, T.; Weiss, G. The struggle for iron-A metal at the host-pathogen interface. Cell. Microbiol. 2010, 12, 1691-1702.

87. Coale, K.H.; Johnson, K.S.; Fitzwater, S.E.; Gordon, R.M.; Tanner, S.; Chavez, F.P.; Ferioli, L.; Sakamoto, C.; Rogers, P.; Millero, F.; et al. A massive phytoplankton bloom induced by an ecosystem-scale iron fertilization experiment in the equatorial Pacific Ocean. Nature 1996, 383, 495-501.

88. Cornelis, P.; Wei, Q.; Andrews, S.C.; Vinckx, T. Iron homeostasis and management of oxidative stress response in bacteria. Met. Integr. Biomet. Sci. 2011, 3, 540-549.

89. Bagg, A.; Neilands, J.B. Ferric uptake regulation protein acts as a repressor, employing iron (II) as a cofactor to bind the operator of an iron transport operon in Escherichia coli. Biochemistry 1987, 26, 5471-5477.

90. Delany, I.; Spohn, G.; Rappuoli, R.; Scarlato, V. The Fur repressor controls transcription of iron-activated and -repressed genes in Helicobacter pylori. Mol. Microbiol. 2001, 42, 1297-1309.

91. Bereswill, S.; Greiner, S.; van Vliet, A.H.; Waidner, B.; Fassbinder, F.; Schiltz, E.; Kusters, J.G.; Kist, M. Regulation of ferritin-mediated cytoplasmic iron storage by the ferric uptake regulator homolog (Fur) of Helicobacter pylori. J. Bacteriol. 2000, 182, 5948-5953.

92. Fillat, M.F. The FUR (ferric uptake regulator) superfamily: Diversity and versatility of key transcriptional regulators. Arch. Biochem. Biophys. 2014, 546, 41-52.

93. Dian, C.; Vitale, S.; Leonard, G.A.; Bahlawane, C.; Fauquant, C.; Leduc, D.; Muller, C.; de Reuse, H.; Michaud-Soret, I.; Terradot, L. The structure of the Helicobacter pylori ferric uptake regulator Fur reveals three functional metal binding sites. Mol. Microbiol. 2011, 79, 1260-1275.

94. Pohl, E.; Haller, J.C.; Mijovilovich, A.; Meyer-Klaucke, W.; Garman, E.; Vasil, M.L. Architecture of a protein central to iron homeostasis: Crystal structure and spectroscopic analysis of the ferric uptake regulator. Mol. Microbiol. 2003, 47, 903-915.

95. Tiss, A.; Barre, O.; Michaud-Soret, I.; Forest, E. Characterization of the DNA-binding site in the ferric uptake regulator protein from Escherichia coli by UV crosslinking and mass spectrometry. FEBS Lett. 2005, 579, 5454-5460.

96. Iwig, J.S.; Chivers, P.T. Coordinating intracellular nickel-metal-site structure-function relationships and the NikR and RcnR repressors. Nat. Prod. Rep. 2010, 27, 658-667.

97. De Pina, K.; Desjardin, V.; Mandrand-Berthelot, M.A.; Giordano, G.; Wu, L.F. Isolation and characterization of the nikR gene encoding a nickel-responsive regulator in Escherichia coli. J. Bacteriol. 1999, 181, 670-674.

98. Chivers, P.T.; Sauer, R.T. Regulation of high affinity nickel uptake in bacteria. $\mathrm{Ni}^{2+}$-Dependent interaction of NikR with wild-type and mutant operator sites. J. Biol. Chem. 2000, 275, 19735-19741.

99. Van Vliet, A.H.; Ernst, F.D.; Kusters, J.G. NikR-mediated regulation of Helicobacter pylori acid adaptation. Trends Microbiol. 2004, 12, 489-494.

100. Musiani, F.; Bertoša, B.; Magistrato, A.; Zambelli, B.; Turano, P.; Losasso, V.; Micheletti, C.; Ciurli, S.; Carloni, P. Computational study of the DNA-binding protein Helicobacter pylori NikR: The role of $\mathrm{Ni}^{2+}$. J. Chem. Theory Comput. 2010, 6, 3503-3515.

101. West, A.L.; St. John, F.; Lopes, P.E.M.; MacKerell, A.D.; Pozharski, E.; Michel, S.L.J. Holo-Ni(II) $H p N i k R$ Is an asymmetric tetramer containing two different nickel-binding sites. J. Am. Chem. Soc. 2010, 132, 14447-14456. 
102. Benini, S.; Cianci, M.; Ciurli, S. Holo-Ni ${ }^{2+}$ Helicobacter pylori NikR contains four square-planar nickel-binding sites at physiological pH. Dalton Trans. 2011, 40, 7831-7833.

103. Dian, C.; Schauer, K.; Kapp, U.; McSweeney, S.M.; Labigne, A.; Terradot, L. Structural basis of the nickel response in Helicobacter pylori: Crystal structures of $H p N i k R$ in apo and nickel-bound states. J. Mol. Biol. 2006, 361, 715-730.

104. Chivers, P.T.; Sauer, R.T. NikR is a ribbon-helix-helix DNA-binding protein. Protein Sci. 1999, 8, 2494-2500.

105. Schreiter, E.R.; Wang, S.C.; Zamble, D.B.; Drennan, C.L. NikR-operator complex structure and the mechanism of repressor activation by metal ions. Proc. Natl. Acad. Sci. USA 2006, 103, 13676-13681.

106. Zambelli, B.; Danielli, A.; Romagnoli, S.; Neyroz, P.; Ciurli, S.; Scarlato, V. High-affinity $\mathrm{Ni}^{2+}$ binding selectively promotes binding of Helicobacter pylori NikR to its target urease promoter. J. Mol. Biol. 2008, 383, 1129-1143.

107. Bahlawane, C.; Dian, C.; Muller, C.; Round, A.; Fauquant, C.; Schauer, K.; de Reuse, H.; Terradot, L.; Michaud-Soret, I. Structural and mechanistic insights into Helicobacter pylori NikR activation. Nucleic Acids Res. 2010, 38, 3106-3118.

108. West, A.L.; Evans, S.E.; Gonzalez, J.M.; Carter, L.G.; Tsuruta, H.; Pozharski, E.; Michel, S.L. $\mathrm{Ni}(\mathrm{II})$ coordination to mixed sites modulates DNA binding of $H p$ NikR via a long-range effect. Proc. Natl. Acad. Sci. USA 2012, 109, 5633-5638.

109. Bradley, M.J.; Chivers, P.T.; Baker, N.A. Molecular dynamics simulation of the Escherichia coli NikR protein: Equilibrium conformational fluctuations reveal interdomain allosteric communication pathways. J. Mol. Biol. 2008, 378, 1155-1173.

110. Cui, G.; Merz, K.M., Jr. The intrinsic dynamics and function of nickel-binding regulatory protein: Insights from elastic network analysis. Biophys. J. 2008, 94, 3769-3778.

111. Mazzei, L.; Dobrovolska, O.; Musiani, F.; Zambelli, B.; Ciurli, S. On the interaction of Helicobacter pylori NikR, a Ni(II)-responsive transcription factor, with the urease operator: In solution and in silico studies. J. Biol. Inorg. Chem. 2015, doi:10.1007/s00775-015-1284-0.

112. Thompson, J.D.; Higgins, D.G.; Gibson, T.J. CLUSTAL W: Improving the sensitivity of progressive multiple sequence alignment through sequence weighting, position-specific gap penalties and weight matrix choice. Nucleic Acids Res. 1994, 22, 4673-4680.

113. Lee, T.I.; Young, R.A. Transcriptional regulation and its misregulation in disease. Cell 2013, 152, 1237-1251.

114. Jubb, H.; Higueruelo, A.P.; Winter, A.; Blundell, T.L. Structural biology and drug discovery for protein-protein interactions. Trends Pharmacol. Sci. 2012, 33, 241-248.

115. Janin, J.; Bonvin, A.M. Protein-protein interactions. Curr. Opin. Struct. Biol. 2013, 23, 859-861.

116. London, N.; Raveh, B.; Schueler-Furman, O. Druggable protein-protein interactions-From hot spots to hot segments. Curr. Opin. Chem. Biol. 2013, 17, 952-959.

117. Milroy, L.G.; Grossmann, T.N.; Hennig, S.; Brunsveld, L.; Ottmann, C. Modulators of protein-protein interactions. Chem. Rev. 2014, 114, 4695-4748.

118. Aeluri, M.; Chamakuri, S.; Dasari, B.; Guduru, S.K.; Jimmidi, R.; Jogula, S.; Arya, P. Small molecule modulators of protein-protein interactions: Selected case studies. Chem. Rev. 2014, 114, 4640-4694. 
119. Wells, J.A.; McClendon, C.L. Reaching for high-hanging fruit in drug discovery at protein-protein interfaces. Nature 2007, 450, 1001-1009.

120. Blundell, T.L.; Burke, D.F.; Chirgadze, D.; Dhanaraj, V.; Hyvonen, M.; Innis, C.A.; Parisini, E.; Pellegrini, L.; Sayed, M.; Sibanda, B.L. Protein-protein interactions in receptor activation and intracellular signalling. Biol. Chem. 2000, 381, 955-959.

121. Koehler, A.N. A complex task? Direct modulation of transcription factors with small molecules. Curr. Opin. Chem. Biol. 2010, 14, 331-340.

122. Nair, S.K.; Burley, S.K. X-ray structures of Myc-Max and Mad-Max recognizing DNA. Molecular bases of regulation by proto-oncogenic transcription factors. Cell 2003, 112, 193-205.

123. Berg, T. Inhibition of transcription factors with small organic molecules. Curr. Opin. Chem. Biol. 2008, 12, 464-471.

124. Bogan, A.A.; Thorn, K.S. Anatomy of hot spots in protein interfaces. J. Mol. Biol. 1998, 280, $1-9$.

125. Blundell, T.L.; Sibanda, B.L.; Montalvao, R.W.; Brewerton, S.; Chelliah, V.; Worth, C.L.; Harmer, N.J.; Davies, O.; Burke, D. Structural biology and bioinformatics in drug design: Opportunities and challenges for target identification and lead discovery. Philos. Trans. R. Soc. B Biol. Sci. 2006, 361, 413-423.

126. Fletcher, S.; Hamilton, A.D. Protein surface recognition and proteomimetics: Mimics of protein surface structure and function. Curr. Opin. Chem. Biol. 2005, 9, 632-638.

(C) 2015 by the authors; licensee MDPI, Basel, Switzerland. This article is an open access article distributed under the terms and conditions of the Creative Commons Attribution license (http://creativecommons.org/licenses/by/4.0/). 